\title{
Non-communicable diseases among adolescents: current status, determinants, interventions and policies
}

\author{
N. Akseer ${ }^{1,2^{*}}$ D, S. Mehta ${ }^{1}$, J. Wigle ${ }^{1,2}$, R. Chera' ${ }^{1}$, Z. J. Brickman', S. Al-Gashm', B. Sorichetti ${ }^{1,2}$, A. Vandermorris ${ }^{1,3}$, \\ D. B. Hipgrave ${ }^{4}$ N. Schwalbe ${ }^{5}$ and Z. A. Bhutta, ${ }^{1,2,6}$
}

\begin{abstract}
Background: Addressing non-communicable disease (NCDs) is a global priority in the Sustainable Development Goals, especially for adolescents. However, existing literature on NCD burden, risk factors and determinants, and effective interventions and policies for targeting these diseases in adolescents, is limited. This study develops an evidence-based conceptual framework, and highlights pathways between risk factors and interventions to NCD development during adolescence (ages 10-19 years) and continuing into adulthood. Additionally, the epidemiologic profile of key NCD risk factors and outcomes among adolescents and preventative NCD policies/ laws/legislations are examined, and a multivariable analysis is conducted to explore the determinants of NCDs among adolescents and adults.
\end{abstract}

Methods: We reviewed literature to develop an adolescent-specific conceptual framework for NCDs. Global data repositories were searched from Jan-July 2018 for data on NCD-related risk factors, outcomes, and policy data for 194 countries from 1990 to 2016. Disability-Adjusted Life Years were used to assess disease burden. A hierarchical modeling approach and ordinary least squares regression was used to explore the basic and underlying causes of NCD burden.

Results: Mental health disorders are the most common NCDs found in adolescents. Adverse behaviours and lifestyle factors, specifically smoking, alcohol and drug use, poor diet and metabolic syndrome, are key risk factors for NCD development in adolescence. Across countries, laws and policies for preventing NCD-related risk factors exist, however those targeting contraceptive use, drug harm reduction, mental health and nutrition are generally limited. Many effective interventions for NCD prevention exist but must be implemented at scale through multisectoral action utilizing diverse delivery mechanisms. Multivariable analyses showed that structural/macro, community and household factors have significant associations with NCD burden among adolescents and adults.

Conclusions: Multi-sectoral efforts are needed to target NCD risk factors among adolescents to mitigate disease burden and adverse outcomes in adulthood. Findings could guide policy and programming to reduce NCD burden in the sustainable development era.

Keywords: Adolescents, Non-communicable diseases, Determinants, Policies, Risk factors

\footnotetext{
* Correspondence: nadia.akseer@gmail.com

'Centre for Global Child Health, Hospital for Sick Children, Toronto, ON M5G OA4, Canada

${ }^{2}$ Dalla Lana School of Public Health, University of Toronto, Toronto, Canada

Full list of author information is available at the end of the article
}

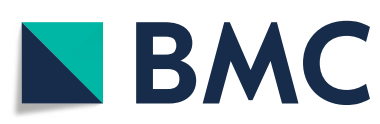

(- The Author(s). 2020 Open Access This article is licensed under a Creative Commons Attribution 4.0 International License, which permits use, sharing, adaptation, distribution and reproduction in any medium or format, as long as you give appropriate credit to the original author(s) and the source, provide a link to the Creative Commons licence, and indicate if changes were made. The images or other third party material in this article are included in the article's Creative Commons licence, unless indicated otherwise in a credit line to the material. If material is not included in the article's Creative Commons licence and your intended use is not permitted by statutory regulation or exceeds the permitted use, you will need to obtain permission directly from the copyright holder. To view a copy of this licence, visit http://creativecommons.org/licenses/by/4.0/. The Creative Commons Public Domain Dedication waiver (http://creativecommons.org/publicdomain/zero/1.0/) applies to the data made available in this article, unless otherwise stated in a credit line to the data. 


\section{Background}

The global burden of non-communicable diseases (NCDs) is a growing public health crisis that requires attention and action from the international community [1]. As the leading cause of mortality, this class of diseases is responsible for 38 million of 57 million annual deaths, with $85 \%$ of these deaths occurring in low- and middle-income countries (LMICs) [2]. However, the discussion on efforts to address NCDs to-date has focused on the adult population, with adolescents largely overlooked [3]. Since NCDs are often considered 'lifestyle illnesses' and the youth population is commonly thought of as healthy, modest efforts have been made to assess their health, disease prevention and lifestyle modification. Yet, adolescents experience a substantial share of the global NCD burden $[4,5]$.

Extensive research has shown that NCDs are primarily attributed to underlying and modifiable risk factors that often emerge during these earlier years [5-7]. It is estimated that approximately $70 \%$ of premature deaths occurring during adulthood are the result of health-related behaviours that are initiated in childhood and adolescence $[3,8]$. Such risk factors, including overweight and obesity, physical inactivity, substance use and poor nutrition, substantially contribute to disease development and poor health in later life [8]. For example, the prevalence of overweight and obesity increases drastically during mid-adolescence and into adulthood [9]. Overweight and obesity during childhood and adolescence represents a significant risk for premature mortality and physical morbidity later in life, including cardiovascular disease, asthma, and certain types of cancers [10]. Drug and substance use also represent a threat for multiple health outcomes, including poor mental health [11]. Since health behaviours and risk exposures that emerge during adolescence underpin health and well-being across the life-course and also affect pregnancy outcomes, investments must be made in the health of current and future generations. In fact, the 2016 Lancet Commission on Adolescent Health and Well-being recommended investment in dominant NCD-related health behaviours among adolescents as a means of preventing future disease development [12].

Addressing NCDs has emerged as a global priority in the Sustainable Development Goals (target 3.4) [13] and the focus of a third UN high-level meeting [14], and includes the establishment and promotion of cost-effective interventions to prevent and address NCDs [15]. However, existing literature provides patchy insight into the current state of NCD-related lifestyle and behavioral risk factors among young people. In fact, to-date, there is no systematic assessment of the NCD burden, risk factors and determinants of NCDs, and effective interventions and policies for targeting these diseases in this population. Further, recent and ongoing efforts by the World Health Organization have involved the establishment of several global strategies, policies, laws and legislations to reduce the harmful use of alcohol [16], increase monitoring and decrease tobacco use [17], as well as to address childhood obesity [18]. However, limited evidence is available on the monitoring and evaluation, and effective implementation of these NCDrelated efforts.

We conducted a comprehensive assessment of NCDs among adolescents, with the specific objective to: 1) develop an evidence-based conceptual framework explaining the determinants, pathways and interventions for NCDs among this age group and in later life; 2) describe the age- and sex-specific burden of major NCDs and risk factors among adolescents, globally and by geographical region; 3) examine major risk factors for adolescent NCDs and their role in adulthood NCD burden; 4) synthesize and summarize available evidence on effective interventions and delivery platforms to reduce the burden of NCDs among adolescents; 5) explore the availability of related laws and policies to reduce NCDs among adolescents globally and by geographical region; and 6) conduct multivariable assessment of key distal and intermediary contextual determinants of NCD morbidity and mortality among adolescents and, separately, the effect of these factors on adulthood NCD burden.

\section{Methods}

We used a life-course approach and socio-ecological model to inform the development of a conceptual framework illustrating factors affecting NCDs in adolescence and in later life. We explored online databases for information pertaining to the burden of NCDs among adolescents, their determinants and risk factors, and relevant policies/interventions for NCDs in this population. Details are included in the Technical Additional file 1.

The proposed conceptual framework integrated several existing models and frameworks, including the $\mathrm{WHO}$ global monitoring framework [19], NCD framework for action/monitoring [20], socio-ecological models of adolescent health and development $[2,21]$ and key frameworks and determinants of adolescent health outlined in the 2012 and 2016 Lancet series on adolescent health [3, 20, 22]. The layout of the conceptual framework was adapted from the model on the life course approach to NCD prevention by WHO [20] and the multi-sectoral nutrition conceptual framework [23, 24]. An iterative process was conducted to identify, integrate and synthesize the concepts, development and structure of the conceptual framework.

Similar approaches were used to collect information on effective NCD-related interventions and laws/legislations/policies available for the prevention of these diseases during adolescence and in later life. The WHO 
Maternal, Newborn, Child and Adolescent Health (MNCAH) policy indicator database [25] contains data on eleven adolescent NCD-related risk and health outcome policies, laws and regulations from 104 low and middle income countries (LMICs) globally. We obtained and analyzed this data; details in the Technical Additional file 1.

We obtained national estimates on health and contextual indicators related to NCD development in adolescents and adults through a review of global data repositories performed from Jan 1st 2018 to July 6, 2018. We focused on 194 countries and assembled panel datasets from 1990 to 2015; we focused on this period for quantitative analysis to evaluate change and distribution across the Millennium Development Goal (MDG) period. Key areas of interest included distal factors (conflict, governance, population density, environment, urbanization, national wealth, health spending, telecommunications, infrastructure), factors intermediary to NCD outcomes (income inequality, women's empowerment, health care services, youth empowerment, socioeconomic status), and proximal factors (i.e. behavioural, biological, nutritional and environmental risk factors). Primary data sources included the Global Health Observatory Data Repository [26], the State of the World's Children global statistics database [27], the World Bank database [28], the United Nations Statistics Division [29] and the 2015 Global Burden of Disease (GBD) study housed at the Institute for Health Metrics and Evaluation (IHME) [30]. Estimates were obtained directly from the respective sources for each indicator without any manipulation. A summary of the hierarchical levels, domains and indicators with sources linked to NCD outcomes among adolescents and later adulthood in this study are included in the appendix (Additional file 1).

The major non-communicable diseases among adolescents were identified using disability-adjusted life years (DALY) data from the GBD. We constructed ranked estimates using national cause-specific DALYs across the six WHO regions (i. African region; ii. region of the Americas; iii. Eastern Mediterranean region; iv. European region; v. South East Asia region, and vi. Western Pacific region), two adolescent age categories (10-14 years, 15-19 years of age) and by sex. We also tabulated the NCD DALYS in late adolescence to adulthood (age 15-49 years, 50-69 years) that are attributable to lifestyle and behavioral risk factors that begin in the critical adolescence period.

We conducted two sets of multivariable analyses to understand the major contextual, social, economic and environmental determinants of NCD-related mortality and morbidity among 1 ) adolescents and 2) adults. This country-level ecological analysis used data for 195 countries obtained from the GBD data repository for the year 2015. For the adolescent model, the primary outcome was the country's NCD DALY rates among 10-19 year old regressed onto a series of fixed-effect covariates. For the adult model, the outcome was country-level NCD DALY rates among 25-59 year olds which was regressed onto a series of contextual, social, economic and environmental factors. We could not run regression models of panel data or change from 1990 to 2015 since many countries did not have reliable estimates for key covariate indicators in 1990. We used a hierarchical modeling approach [20] with structural, national, community, household and individual level covariate indicators mapped to the respective levels described above (Additional file 1). We selected largely distal and intermediary factors that could have a differential impact on proximal risk factors of NCD development in adolescence and in later adulthood. Ordinary least squares regression models were fitted, and crude and standardized beta coefficients were estimated. Variables with skewed distributions were transformed appropriately to stabilize variance for regression analysis. Variables associated with the outcome at $p<0.20$ in bivariate analysis were entered into multivariable model selection algorithms. Elastic net regression was used to select statistically significant variables $(p<0.15)$ in multivariable analysis at each level due to the large number of correlated predictors. Collinearity was assessed using variance inflation factors, and residual plots, influence/deviance statistics, AIC/BIC and adjusted R2 were consulted to assess model fit. Type 1 error rate was retained at 0.05 and analyses were conducted using SAS version 9.4 and RStudio Version 1.1.423.

\section{Results}

\section{Conceptual framework}

Our novel evidence-based conceptual framework (Fig. 1) represents a comprehensive and integrated approach to understanding the complex pathways through which risk and protective factors contribute to NCDs among adolescents and in later life. The framework provides a depiction of four key components: i) societal determinants; ii) individual predisposing factors across the adolescent and young adulthood stages of the life course; iii) top NCD conditions among adolescents and in later adulthood; and iv) select evidence-based policies, laws and interventions for this population. Key definitions, pathways and relationships of the NCD conceptual framework are detailed in Table 1 [31-35, 37].

\section{Burden and trends of NCD outcomes in adolescents}

We explored the current burden of NCDs among adolescents by age and sex (Table 2), as well as by WHO region (Additional file 1). A large share of the burden of NCDs among 10-19 year olds is due to mental illnesses. Conduct disorder accounts for the most NCD DALYs $(2595,245)$ among adolescents aged $10-14$, representing 

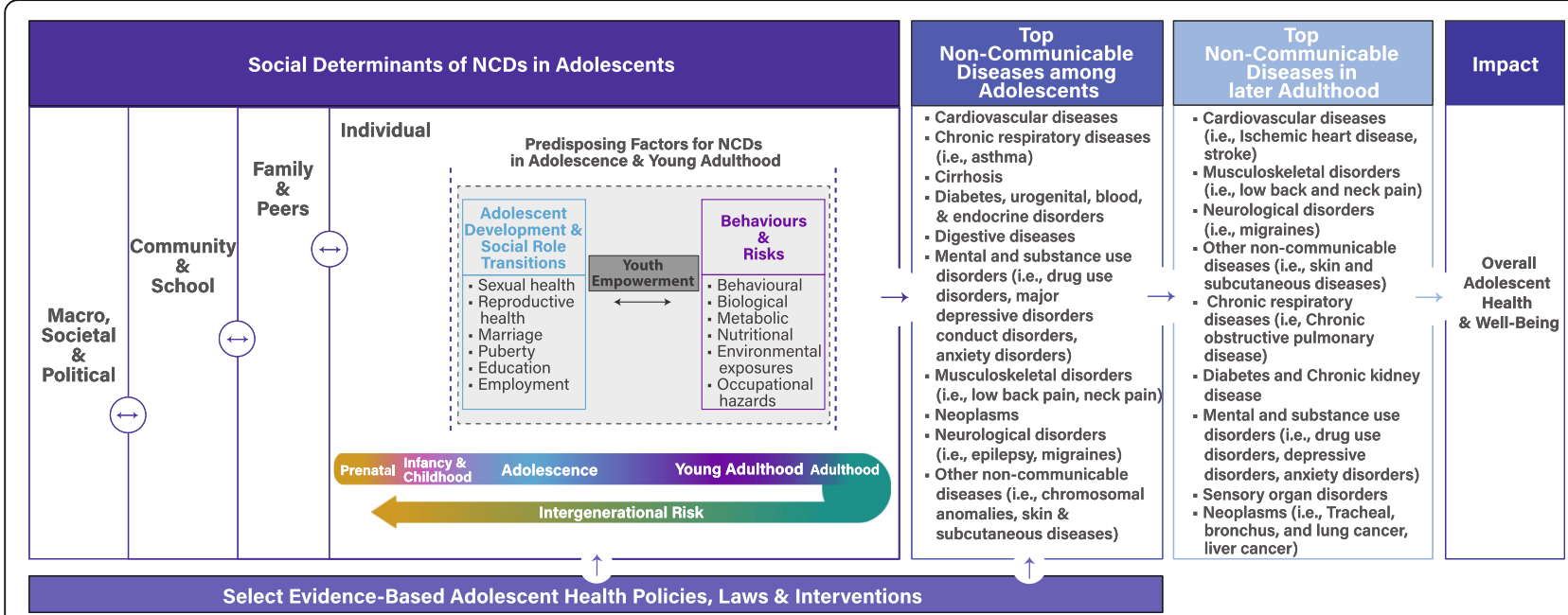

Fig. 1 Conceptual framework on NCDs among adolescents

$8 \%$ of the total NCD burden in this age group. In addition, anxiety disorders $(2,100,974 ; 6 \%)$ and major depressive disorder $(1,472,928 ; 4 \%)$ were the 3rd and 6th highest ranked NCD cause of DALYs among 10-14 yearolds, respectively. In 15-19 year olds, major depressive disorder is the top NCD cause of DALYs (3646,293), representing $8 \%$ of the total NCD burden in this age group. Comparable to the 10-14-year-old age group, anxiety disorders $(2,510,537 ; 6 \%)$ and conduct disorder (1874, 317 ; 4\%) ranked 3rd and 7th, respectively, in the rank of top 10 NCDs with the highest DALY burden (Table 2).

DALYs due to NCD causes also vary by sex (Table 2 ). In 10-14 year olds, conduct disorder contributes the most NCD DALYs for males (1638,150; 9\%), followed by asthma (1204,058), anxiety disorders $(838,606)$, migraine $(773,026)$ and acne vulgaris $(722,333)$. In females of the same age, migraine contributes the greatest burden of disease $(1273,422 ; 8 \%)$, followed by anxiety disorders $(1262,368)$, asthma $(1117,023)$, conduct disorder (957, 096) and major depressive disorder $(825,013)$. In 15-19 year olds, major depressive disorder contributes the most NCD DALYs for both males $(1,588,775)$ and females $(2,057,518)$; however, it does represent a greater proportion of all DALYs in females (9\%) than in males (7\%) in this age group. Overall, in both adolescent age groups, conduct disorder plays a larger role in males than females, and anxiety disorders and major depressive disorders play a larger role in females.

Across global regions, conduct disorder persists as the leading NCD among males 10-14 years of age (Additional file 1). In females of the same age, migraines are the most prominent NCD in the Eastern Mediterranean and South East Asia regions, anxiety disorders are the most prominent in the Americas and European and Western Pacific regions, and asthma is the most prominent NCD among girls in
Africa. Among males 15-19 years of age, major depressive disorder is the leading NCD in Africa, the Americas, the Eastern Mediterranean, and South East Asia, whereas low back pain and acne vulgaris are the leading NCDs in Europe and in the Western Pacific respectively. Similarly, among females 15-19 years of age, depression contributes the most NCD DALYs in four of the six regions (Africa, Europe, Americas, Western Pacific), whereas migraine leads in the Eastern Mediterranean and South East Asia regions (Additional file 1).

\section{Burden, Behavioural, and socioeconomic risk factors}

Select behavioural, lifestyle and socioeconomic risk factors for NCDs from adolescence into adulthood in regions around the world are highlighted in Table 3. With regards to lifestyle and behavioural factors, the prevalence of alcohol drinking among 15-19 year olds is more common among boys than girls, and is highest in regions of the Americas and Europe (>50\%). Similarly, tobacco use among young adolescents is higher among males compared to females, and ranges from 12 to $2 \%$ across regions. Insufficient physical activity is pervasive in almost $80 \%$ or more of adolescents aged 11-17 years across all regions and both sexes. The prevalence of overweight children and adolescents under 20 years of age is higher among males compared to females across all regions, the region with largest rate for both genders was the Americas with more than a third of the under 20 population being classified as overweight. Obesity in children and adolescents under 20 years of age is also higher among males and is highest in the regions of Middle East and North Africa and Europe. Youth literacy rates are high (almost $90 \%$ or more) in most regions but lag behind in Africa and South Asia. Secondary 
Table 1 Conceptual framework for NCDs among adolescents

The evidence-based conceptual framework (Fig. 1) represents a comprehensive, integrated and novel approach to understanding the complex pathways through which structural, community, school, family, peer, and individual risk and protective factors contribute to the global burden of NCDs among adolescents and in later adulthood.

\section{Key definitions}

Social Determinants of Health: Conditions in which people are born, grow, develop, live, work and age. This includes the social, political, economic, environmental and cultural systems and forces that influence and shape the health and wellbeing of individuals.

Macro, Societal \& Political: Fundamental global and national social, economic, and political structures that shape inequalities in society, health and individual development. Macro trends include factors such as migration, conflict, environmental conditions, global economic development, technology and innovation. Societal and political determinants also represent underlying factors that substantially impact health, including national wealth, policies and laws, infrastructure, population density, governance, and culture.

Community \& School: Circumstances of daily life where adolescents live, work and learn, including youth and women's empowerment, quality and access to health services, education, transportation, access to health services and employment opportunities and conditions.

Family \& Peer Factors: The primary protective and enabling structures that shape the health and development of young people. Relationships, connectedness, and the behaviours of family (e.g. parents, guardians, caretakers and siblings) and peers significantly influence the behaviour and health of adolescents.

Individual Factors: Individual developmental milestones and social role transitions, including sexual and reproductive health, early marriage, age of puberty, education and employment. Behavioural, biological, metabolic, nutritional, environmental and occupational factors that are protective or risk factors for individual adolescent health.

Macro, societal and political factors, including climate change and natural disasters, conflict, national wealth and health spending, infrastructure and urbanization, and governance represent critical overarching influences that shape the development and health of adolescents globally. These factors underlie and influence community and school factors, for example urbanization can improve young people's access to education and health services, however may also increase young people's risk for NCD-related risk factors, including mental health issues and obesity and physical inactivity [12]. Community and school level determinants play a substantial role in determining the current and future health of adolescents. Income inequality is associated with overall health outcomes, including mortality rates $[9,13]$ Inequalities in socioeconomic status also represent risks for NCDs including, increased physical inactivity, high BMI, poor psychological and physical well-being, high rates of substance use, bullying, and other poor behavioural and health outcomes [31, 32]. Access to education and educational attainment represent critical protective factors for health across the life-course. Better-educated individuals live longer and healthier lives globally, with lower mortality, and improved self-reported health outcomes [22, 33]. Diverse adolescent health outcomes and behaviours, including sexual and reproductive health, child marriage, mental health and self-harm [34] and obesity [33], are all positively influenced by increased educational attainment, particularly completion of secondary education. In addition, education significantly shapes the health of future generations, empowers youth and women, and narrows inequalities in status and health [35]. Availability and accessibility of health services represents a key approach to addressing and managing chronic health conditions and NCDs [36]. Youth unemployment and low-quality, unsafe, employment opportunities have been identified to significantly impact adolescents' well-being, job satisfaction and health [37], including association with poor mental health, suicide and violence [22].

Family and peer connectedness, modeling of behaviours, and relationships represent significant protective or risk factors for adolescent health behaviours and outcomes, including smoking, violence, suicidal thoughts and behaviours, sexual and reproductive health, and overall healthy development $[2,36]$. Connectedness and attitudes towards school have been associated with substance use, including drug, alcohol and tobacco [36]. Behaviours and risks vary across the life-course, and impact children and adolescents' growth, development and risk for NCDs. Adolescence represents a time of significant biological, developmental and social role changes and transitions, including puberty, sexual and reproductive health, education, marriage, and employment. Furthermore, the initiation of behaviours such as tobacco use, poor diet, physical inactivity, and consumption of alcohol during childhood and adolescence contributes to the burden of disease during this time period, and substantially increases the risks for NCDs in later life. Mitigating and protective factors during adolescence include female empowerment and the empowerment of young people [35, 36]. The improved status of women in society (e.g. education, employment, increased age of marriage, etc.), has been associated with improved health outcomes for children and adolescents, while increased empowerment, education and employment of young people, are related to improved mental and physical health outcomes $[22,33,34,36]$. The risk of developing NCDs increases across the life-course from childhood into adulthood [5], including increased risk of asthma, cardiovascular disease, diabetes, mental and substance use disorders (e.g. drug use disorders, major depressive disorder, etc.), musculoskeletal disorders, cancer, neurological disorders and other NCDs. Peer, family, community, national and broader global social determinants represent complex and interrelated factors that influence and shape individual behaviours and risks contributing to increased burden of NCDs. A supportive and enabling environment to develop and implement policies and interventions targeting structural, community, school, individual and crosscutting levels, represent a critical approach to improving adolescents' health and development, and to addressing health behaviours and causes of NCDs (Appendix 7).

school enrollment rates are also consistent across most regions (70\% or more) except for Eastern and Southern Africa and South Asia regions. Youth literacy and secondary school enrollment rates are similar between genders and this similarity is synonymous across all regions. Unemployment among youth $15-24$ years is highest $(28 \%+)$ in the MENA region followed by the Americas (18.5\%) and Europe regions (18.5\%).Breastfeeding patterns have been linked to overweight, type 2 diabetes, and possibly high blood pressure and cholesterol in childhood and adolescence, and even into adulthood [39, 40]. About 50\% of South Asia and Eastern and
Southern Africa exclusively breastfeeds child younger than 6 months, while only 28 to $39 \%$ of those in other regions practice exclusive breastfeeding. Sanitation is part of the broad set of environmental factors that directly and indirectly influence risk of NCDs in childhood and adolescence. Poor sanitation can result in, for instance, diarrhea, which can impact nutrient absorption and disease burden. It's also linked to individual and social perceptions about health and wellbeing, which can influence an adolescent' mental health [36, 41]. Access to improved water (53-62\%) and improved sanitation (27-30\%) facilities is notably lower in Africa while all other regions have $>80 \%$ availability. 
Table 2 Distribution of NCD DALYs among adolescents by age and sex in 2015

\begin{tabular}{|c|c|c|c|c|c|c|c|c|c|c|}
\hline \multirow[b]{2}{*}{$\begin{array}{l}\text { Age } \\
\text { group }\end{array}$} & \multirow[b]{2}{*}{ Rank } & \multicolumn{3}{|l|}{ Both } & \multicolumn{3}{|l|}{ Male } & \multicolumn{3}{|l|}{ Female } \\
\hline & & Health Outcome & $\begin{array}{l}\text { DALYs } \\
\text { (1000s) }\end{array}$ & $\begin{array}{l}\% \text { of } \\
\text { Total }\end{array}$ & Health Outcome & $\begin{array}{l}\text { Total } \\
\text { DALYs } \\
\text { (1000s) }\end{array}$ & $\begin{array}{l}\% \text { of } \\
\text { Total }\end{array}$ & Health Outcome & $\begin{array}{l}\text { Total } \\
\text { DALYs } \\
\text { (1000s) }\end{array}$ & $\begin{array}{l}\% \text { of } \\
\text { Total }\end{array}$ \\
\hline \multirow{13}{*}{$\begin{array}{l}10-14 \\
\text { years old }\end{array}$} & 1 & Conduct disorder & 2595 & 7.74 & Conduct disorder & 1638 & 9.42 & Migraine & 1273 & 7.89 \\
\hline & 2 & Asthma & 2321 & 6.92 & Asthma & 1204 & 6.92 & Anxiety disorders & 1262 & 7.82 \\
\hline & 3 & Anxiety disorders & 2101 & 6.26 & Anxiety disorders & 839 & 4.82 & Asthma & 1117 & 6.92 \\
\hline & 4 & Migraine & 2046 & 6.10 & Migraine & 773 & 4.44 & Conduct disorder & 957 & 5.93 \\
\hline & 5 & Acne vulgaris & 1476 & 4.40 & Acne vulgaris & 722 & 4.15 & $\begin{array}{l}\text { Major depressive } \\
\text { disorder }\end{array}$ & 825 & 5.11 \\
\hline & 6 & $\begin{array}{l}\text { Major depressive } \\
\text { disorder }\end{array}$ & 1473 & 4.39 & Low back pain & 649 & 3.73 & Acne vulgaris & 753 & 4.67 \\
\hline & 7 & Low back pain & 1270 & 3.79 & $\begin{array}{l}\text { Major depressive } \\
\text { disorder }\end{array}$ & 648 & 3.72 & Low back pain & 621 & 3.85 \\
\hline & 8 & $\begin{array}{l}\text { Age-related and } \\
\text { other hearing loss }\end{array}$ & 1123 & 3.35 & $\begin{array}{l}\text { Age-related and } \\
\text { other hearing loss }\end{array}$ & 626 & 3.60 & $\begin{array}{l}\text { Age-related and } \\
\text { other hearing loss }\end{array}$ & 497 & 3.08 \\
\hline & 9 & Epilepsy & 992 & 2.96 & Epilepsy & 539 & 3.10 & Dermatitis & 482 & 2.98 \\
\hline & 10 & Dermatitis & 906 & 2.70 & Autism & 454 & 2.61 & Epilepsy & 453 & 2.81 \\
\hline & & \multicolumn{2}{|c|}{$\begin{array}{l}\text { Percent of total NCD burden } \\
\text { in age and sex }\end{array}$} & 48.60 & \multicolumn{2}{|c|}{$\begin{array}{l}\text { Percent of total NCD burden in } \\
\text { age and sex }\end{array}$} & 46.51 & \multicolumn{2}{|c|}{$\begin{array}{l}\text { Percent of total NCD burden in } \\
\text { age and sex }\end{array}$} & 51.04 \\
\hline & & NCD DALYs & 33,546 & & NCD DALYs & 17,398 & & NCD DALYS & 16,147 & \\
\hline & & Total DALYs & 70,005 & & Total DALYs & 38,135 & & Total DALYs & 31,870 & \\
\hline $\begin{array}{l}\text { Age } \\
\text { group }\end{array}$ & Rank & Health Outcome & $\begin{array}{l}\text { DALYs } \\
(1000 s)\end{array}$ & $\begin{array}{l}\% \text { of } \\
\text { Total }\end{array}$ & Health Outcome & $\begin{array}{l}\text { Total } \\
\text { DALYs } \\
\text { (1000s) }\end{array}$ & $\begin{array}{l}\% \text { of } \\
\text { Total }\end{array}$ & Health Outcome & $\begin{array}{l}\text { Total } \\
\text { DALYs } \\
\text { (1000s) }\end{array}$ & $\begin{array}{l}\% \text { of } \\
\text { Total }\end{array}$ \\
\hline \multirow{13}{*}{$\begin{array}{l}15-19 \\
\text { Years } \\
\text { Old }\end{array}$} & 1 & $\begin{array}{l}\text { Major depressive } \\
\text { disorder }\end{array}$ & 3646 & 8.16 & $\begin{array}{l}\text { Major depressive } \\
\text { disorder }\end{array}$ & 1589 & 6.98 & $\begin{array}{l}\text { Major depressive } \\
\text { disorder }\end{array}$ & 2058 & 9.38 \\
\hline & 2 & Migraine & 2709 & 6.06 & Low back pain & 1323 & 5.81 & Migraine & 1673 & 7.63 \\
\hline & 3 & Anxiety disorders & 2511 & 5.62 & Conduct disorder & 1232 & 5.41 & Anxiety disorders & 1518 & 6.92 \\
\hline & 4 & Low back pain & 2478 & 5.54 & Acne vulgaris & 1065 & 4.68 & Low back pain & 1155 & 5.27 \\
\hline & 5 & Acne vulgaris & 2151 & 4.81 & Migraine & 1036 & 4.55 & Acne vulgaris & 1086 & 4.95 \\
\hline & 6 & $\begin{array}{l}\text { Other } \\
\text { musculoskeletal } \\
\text { disorders }\end{array}$ & 1923 & 4.30 & Anxiety disorders & 993 & 4.36 & $\begin{array}{l}\text { Other } \\
\text { musculoskeletal } \\
\text { disorders }\end{array}$ & 1051 & 4.79 \\
\hline & 7 & Conduct disorder & 1874 & 4.19 & Asthma & 889 & 3.90 & Asthma & 865 & 3.95 \\
\hline & 8 & Asthma & 1755 & 3.92 & $\begin{array}{l}\text { Other } \\
\text { musculoskeletal } \\
\text { disorders }\end{array}$ & 872 & 3.83 & Conduct disorder & 643 & 2.93 \\
\hline & 9 & Epilepsy & 1150 & 2.57 & Epilepsy & 682 & 3.00 & $\begin{array}{l}\text { Age-related and } \\
\text { other hearing loss }\end{array}$ & 486 & 2.21 \\
\hline & 10 & $\begin{array}{l}\text { Age-related and } \\
\text { other hearing loss }\end{array}$ & 1123 & 2.51 & $\begin{array}{l}\text { Age-related and } \\
\text { other hearing loss }\end{array}$ & 638 & 2.80 & Epilepsy & 468 & 2.13 \\
\hline & & \multicolumn{2}{|c|}{$\begin{array}{l}\text { Percent of total NCD burden } \\
\text { in age and sex }\end{array}$} & 47.69 & \multicolumn{2}{|c|}{$\begin{array}{l}\text { Percent of total NCD burden in } \\
\text { age and sex }\end{array}$} & 45.32 & \multicolumn{2}{|c|}{$\begin{array}{l}\text { Percent of total NCD burden in } \\
\text { age and sex }\end{array}$} & 50.16 \\
\hline & & NCD DALYs & 44,706 & & NCD DALYs & 22,771 & & NCD DALYS & 21,935 & \\
\hline & & Total DALYs & 85,151 & & Total DALYs & 46,335 & & Total DALYs & 38,816 & \\
\hline
\end{tabular}

Death and disability are associated with risk factors that begin in adolescence, although causality cannot be inferred from these data (Additional file 1). The top causes of premature death among 15-49 year olds are related to high body mass index (e.g. higher body adiposity), high blood pressure, high plasma blood glucose (diabetes), high cholesterol, smoking, drug and alcohol use, diets low in fruits and whole grains, and air 
Table 3 Distribution of select NCD risk factors among adolescents by global region

\begin{tabular}{|c|c|c|c|c|c|c|c|c|c|c|c|}
\hline \multicolumn{2}{|c|}{ Risk Factor Indicators } & \multirow[t]{2}{*}{ Sex } & \multicolumn{7}{|l|}{ Regions $^{1}$} & \multirow{2}{*}{$\begin{array}{l}\text { Data } \\
\text { Source }\end{array}$} & \multirow{2}{*}{$\begin{array}{l}\text { Year of } \\
\text { Data } \\
\text { Collection }\end{array}$} \\
\hline & & & Americas & Europe & $\begin{array}{l}\text { Eastern } \\
\text { and } \\
\text { Southern } \\
\text { Africa }\end{array}$ & $\begin{array}{l}\text { Western } \\
\text { and } \\
\text { Central } \\
\text { Africa }\end{array}$ & $\begin{array}{l}\text { Middle } \\
\text { East \& } \\
\text { North } \\
\text { Africa }\end{array}$ & Pacific & $\begin{array}{l}\text { South } \\
\text { Asia }\end{array}$ & & \\
\hline \multirow{9}{*}{$\begin{array}{l}\text { Behavioural } \\
\text { Risk Factors }\end{array}$} & \multirow{2}{*}{$\begin{array}{l}\text { Prevalence of current } \\
\text { adolescent drinkers aged } \\
15-19 \text { (\%) [26] }\end{array}$} & Males & 54.9 & 69 & 38.5 & 34.5 & 14.1 & 25.2 & 9 & \multirow[t]{2}{*}{ WHO } & \multirow[t]{2}{*}{2010} \\
\hline & & Females & 37.7 & 48.7 & 25.9 & 22.2 & 11.1 & 15.3 & 4.3 & & \\
\hline & \multirow{2}{*}{$\begin{array}{l}\text { Prevalence of current } \\
\text { smokers of cigarettes } \\
\text { aged } 13-15 \text { per } 100 \\
\text { population (\%) [26] }\end{array}$} & Males & 17.4 & 14.5 & 7.4 & 7.3 & 10.2 & 9.9 & 5.1 & \multirow[t]{2}{*}{ WHO } & \multirow{2}{*}{$\begin{array}{l}2008- \\
2010\end{array}$} \\
\hline & & Females & 19.1 & 9.9 & 3.2 & 2 & 2.4 & 1.9 & 2 & & \\
\hline & \multirow{2}{*}{$\begin{array}{l}\text { Prevalence of current } \\
\text { tobacco use among } \\
\text { adolescents aged 13-15 } \\
\text { years (\%) [26] }\end{array}$} & Males & 17 & - & - & - & 21.3 & 12.4 & 21 & \multirow[t]{2}{*}{ WHO } & \multirow{2}{*}{$\begin{array}{l}2007- \\
2014\end{array}$} \\
\hline & & Females & 13.8 & - & - & - & 9.7 & 3.5 & 7.4 & & \\
\hline & \multirow{3}{*}{$\begin{array}{l}\text { Prevalence of insufficient } \\
\text { physical activity (school- } \\
\text { going adolescents 11-17 } \\
\text { years) [26] }\end{array}$} & $\begin{array}{l}\text { Both } \\
\text { Sexes }\end{array}$ & 81.2 & 83.2 & $85.2^{*}$ & & 87.5 & 85 & 73.4 & \multirow[t]{3}{*}{ WHO } & \multirow[t]{3}{*}{2010} \\
\hline & & Males & 87.1 & 87.7 & $87.9^{*}$ & & 91 & 88.9 & 74.6 & & \\
\hline & & Females & 75.3 & 78.4 & $82.3^{*}$ & & 84.7 & 81 & 72.5 & & \\
\hline $\begin{array}{l}\text { Biological Risk } \\
\text { Factors }\end{array}$ & Low birthweight (\%) [27] & $\begin{array}{l}\text { Both } \\
\text { Sexes }\end{array}$ & 9 & 6 & - & - & - & - & - & UNICEF & $\begin{array}{l}2011- \\
2016\end{array}$ \\
\hline \multirow[t]{11}{*}{$\begin{array}{l}\text { Nutrition Risk } \\
\text { Factors }\end{array}$} & $\begin{array}{l}\text { Early initiation of } \\
\text { breastfeeding (\%) [27] }\end{array}$ & $\begin{array}{l}\text { Both } \\
\text { Sexes }\end{array}$ & 54 & 57 & 63 & 40 & 40 & 43 & 39 & UNICEF & $\begin{array}{l}2011- \\
2016\end{array}$ \\
\hline & $\begin{array}{l}\text { Introduction to solid, } \\
\text { semi-solid or soft foods 6- } \\
8 \text { months (\%) [27] }\end{array}$ & $\begin{array}{l}\text { Both } \\
\text { Sexes }\end{array}$ & 82 & 69 & 75 & 68 & 63 & 69 & 56 & UNICEF & $\begin{array}{l}2011- \\
2016\end{array}$ \\
\hline & $\begin{array}{l}\text { Exclusive breastfeeding }(< \\
6 \text { months, \%) [27] }\end{array}$ & $\begin{array}{l}\text { Both } \\
\text { Sexes }\end{array}$ & 38 & 30 & 55 & 29 & 32 & 28 & 52 & UNICEF & $\begin{array}{l}2011- \\
2016\end{array}$ \\
\hline & $\begin{array}{l}\text { Underweight, moderate } \\
\text { and severe, under-5 (\%) } \\
\text { [26] }\end{array}$ & $\begin{array}{l}\text { Both } \\
\text { Sexes }\end{array}$ & 1.6 & - & $17.2^{*}$ & & 12.8 & 2.7 & 26.2 & WHO & 2017 \\
\hline & $\begin{array}{l}\text { Stunting, moderate and } \\
\text { severe, under-5 (\%) [27] }\end{array}$ & $\begin{array}{l}\text { Both } \\
\text { Sexes }\end{array}$ & 11 & 6 & 34 & 34 & 15 & 9 & 36 & UNICEF & $\begin{array}{l}2011- \\
2016\end{array}$ \\
\hline & $\begin{array}{l}\text { Wasting, moderate and } \\
\text { severe, under-5 (\%) [27] }\end{array}$ & $\begin{array}{l}\text { Both } \\
\text { Sexes }\end{array}$ & 1 & 2 & 7 & 9 & 7 & 3 & 16 & UNICEF & $\begin{array}{l}2011- \\
2016\end{array}$ \\
\hline & $\begin{array}{l}\text { Overweight, moderate } \\
\text { and severe, under-5 (\%) } \\
\text { [27] }\end{array}$ & $\begin{array}{l}\text { Both } \\
\text { Sexes }\end{array}$ & 7 & 13 & 4 & 4 & 11 & 6 & 4 & UNICEF & $\begin{array}{l}2011- \\
2016\end{array}$ \\
\hline & \multirow{2}{*}{$\begin{array}{l}\text { Prevalence of overweight } \\
\text { among children and } \\
\text { adolescents, 5-19years } \\
\text { (\%) [27] }\end{array}$} & Males & 34.6 & 28.1 & - & - & 20.2 & 30.4 & 9.6 & \multirow[t]{2}{*}{ UNICEF } & \multirow[t]{2}{*}{2016} \\
\hline & & Females & 32.6 & 24.2 & - & - & 20.7 & 18.8 & 8.1 & & \\
\hline & \multirow[t]{2}{*}{ Obese, under 20 years (\%) } & Males & 5 & 7.4 & 3.9 & 4.4 & 20.7 & 3.8 & 2.5 & \multirow{2}{*}{$\begin{array}{l}\mathrm{Ng}, \\
2014\end{array}$} & \multirow[t]{2}{*}{2013} \\
\hline & & Females & 4.7 & 6.3 & 4 & 3.2 & 11 & 3.5 & 2.6 & & \\
\hline \multirow{9}{*}{$\begin{array}{l}\text { Socioeconomic } \\
\text { Status }\end{array}$} & \multirow{2}{*}{$\begin{array}{l}\text { Youth literacy rate, aged } \\
15-24 \text { years (\%) [27] }\end{array}$} & Males & 98 & 100 & 87 & 69 & 91 & 99 & 88 & UNICEF & $2011-$ \\
\hline & & Females & 99 & 99 & 85 & 55 & 88 & 97 & 80 & & \\
\hline & Primary school, net & Males & 96 & 94 & 78 & 72 & 94 & 97 & & UNICEF & $2008-$ \\
\hline & attendance ratio (\%) [2/] & Females & 96 & 95 & 79 & 68 & 93 & 97 & & & \\
\hline & Secondary school, net & Males & 74 & 93 & 29 & - & 74 & 71 & 63 & UNICEF & $2011-$ \\
\hline & enrolment ratio (\%) [2/] & Females & 77 & 92 & 30 & - & 74 & 76 & 66 & & \\
\hline & Out-of-school rate of & Males & 5 & 4 & 17 & - & 6 & 6 & 5 & UNICEF & $2011-$ \\
\hline & age (\%) [27] & Females & 4 & 4 & 19 & - & 8 & 6 & 6 & & 2010 \\
\hline & Unemployment, youth & Both & 18.5 & 18.5 & 14.2 & & 28.1 & 10.3 & 10.4 & World & 2017 \\
\hline
\end{tabular}


Table 3 Distribution of select NCD risk factors among adolescents by global region (Continued)

\begin{tabular}{|c|c|c|c|c|c|c|c|c|c|c|c|}
\hline \multicolumn{2}{|c|}{ Risk Factor Indicators } & \multirow[t]{2}{*}{ Sex } & \multicolumn{7}{|l|}{ Regions $^{1}$} & \multirow{2}{*}{$\begin{array}{l}\text { Data } \\
\text { Source }\end{array}$} & \multirow{2}{*}{$\begin{array}{l}\text { Year of } \\
\text { Data } \\
\text { Collection }\end{array}$} \\
\hline & & & Americas & Europe & $\begin{array}{l}\text { Eastern } \\
\text { and } \\
\text { Southern } \\
\text { Africa }\end{array}$ & $\begin{array}{l}\text { Western } \\
\text { and } \\
\text { Central } \\
\text { Africa }\end{array}$ & $\begin{array}{l}\text { Middle } \\
\text { East \& } \\
\text { North } \\
\text { Africa }\end{array}$ & Pacific & $\begin{array}{l}\text { South } \\
\text { Asia }\end{array}$ & & \\
\hline & $\begin{array}{l}\text { total (\% of total labor } \\
\text { force ages 15-24) [28] }\end{array}$ & Sexes & & & & & & & & Bank & \\
\hline & Child labour (\%) [27] & $\begin{array}{l}\text { Both } \\
\text { Sexes }\end{array}$ & 11 & - & 26 & 32 & 7 & - & - & UNICEF & $\begin{array}{l}2010- \\
2016\end{array}$ \\
\hline \multirow{2}{*}{$\begin{array}{l}\text { Access to } \\
\text { Improved } \\
\text { Water and } \\
\text { Sanitation }\end{array}$} & $\begin{array}{l}\text { Improved water, total (\% } \\
\text { of population with access) } \\
{[27]}\end{array}$ & $\begin{array}{l}\text { Both } \\
\text { Sexes }\end{array}$ & 96 & 95 & 53 & 62 & 93 & 94 & 88 & UNICEF & 2015 \\
\hline & $\begin{array}{l}\text { Improved sanitation } \\
\text { facilities, total (\% of } \\
\text { population with access) } \\
{[27]}\end{array}$ & $\begin{array}{l}\text { Both } \\
\text { Sexes }\end{array}$ & 86 & 93 & 30 & 27 & 89 & 77 & 46 & UNICEF & 2015 \\
\hline \multirow{4}{*}{$\begin{array}{l}\text { Health Care } \\
\text { Services and } \\
\text { Essential } \\
\text { Commodities/ } \\
\text { Medicine }\end{array}$} & $\begin{array}{l}\text { Skilled birth attendance, } \\
\text { aged 15-49years (\%) [27] }\end{array}$ & $\begin{array}{l}\text { Both } \\
\text { Sexes }\end{array}$ & 96 & 99 & 60 & 52 & 86 & 95 & 73 & UNICEF & $\begin{array}{l}2013- \\
2016\end{array}$ \\
\hline & $\begin{array}{l}\text { Measles (MCV } \\
\text { immunization on } \\
\text { coverage among } 1 \text { year } \\
\text { olds) (\%) [27] }\end{array}$ & $\begin{array}{l}\text { Both } \\
\text { Sexes }\end{array}$ & 92 & 93 & 76 & 67 & 89 & 93 & 84 & UNICEF & 2016 \\
\hline & $\begin{array}{l}\text { DPT3 immunization } \\
\text { coverage among 1-year } \\
\text { olds (\%) [27] }\end{array}$ & $\begin{array}{l}\text { Both } \\
\text { Sexes }\end{array}$ & 90 & 90 & 80 & 67 & 88 & 94 & 86 & UNICEF & 2016 \\
\hline & $\begin{array}{l}\text { Antenatal care coverage } \\
\text { (4+ visits) (aged 15-49 } \\
\text { years) (\%) [27] }\end{array}$ & Females & 90 & 87 & 52 & 52 & 66 & 74 & 46 & UNICEF & 2016 \\
\hline \multirow[t]{4}{*}{$\begin{array}{l}\text { Fertility Rates/ } \\
\text { Women } \\
\text { Empowerment }\end{array}$} & $\begin{array}{l}\text { Adolescent birth rate, } \\
\text { number of births per } 1000 \\
\text { adolescent girls aged } 15- \\
19 \text { years [27] }\end{array}$ & Females & 74 & 29 & 113 & 130 & 41 & 21 & 44 & UNICEF & $\begin{array}{l}2009- \\
2014\end{array}$ \\
\hline & $\begin{array}{l}\text { Percent of women giving } \\
\text { birth by age } 18(\%)[27]\end{array}$ & Females & 19 & 4 & 26 & 29 & 8 & 7 & 20 & UNICEF & $\begin{array}{l}2011- \\
2016\end{array}$ \\
\hline & $\begin{array}{l}\text { Married or in-union } \\
\text { women of reproductive } \\
\text { age who have their need } \\
\text { for family planning satis- } \\
\text { fied with modern } \\
\text { methods (\%) [26] }\end{array}$ & Females & 83 & 75.1 & $52.2^{*}$ & & 63.6 & 89.7 & 75.1 & WHO & 2018 \\
\hline & $\begin{array}{l}\text { Unmet need for family } \\
\text { planning (\%) (aged 15-49 } \\
\text { years) [26] }\end{array}$ & Females & 9.4 & 10.4 & $24.4^{*}$ & & 17.7 & 5.8 & 13.3 & WHO & 2010 \\
\hline \multirow[t]{3}{*}{$\begin{array}{l}\text { Gender } \\
\text { Inequality }\end{array}$} & $\begin{array}{l}\text { Percent of women aged } \\
20-24 \text { years who were } \\
\text { married by age } 15(\%) \text { [27] }\end{array}$ & Females & & 1 & 9 & 14 & 3 & 2 & & UNICEF & $\begin{array}{l}2010- \\
2016\end{array}$ \\
\hline & $\begin{array}{l}\text { Percent of women aged } \\
20-24 \text { years who were } \\
\text { married by age } 18(\%) \text { [27] }\end{array}$ & Females & & 11 & 35 & 41 & 17 & 15 & & UNICEF & $\begin{array}{l}2010- \\
2016\end{array}$ \\
\hline & $\begin{array}{l}\text { Prevalence of FGM/C (\% } \\
\text { of girls and women of } \\
\text { reproductive age } 15-49 \\
\text { years experiencing FGM/ } \\
\text { C) [27] }\end{array}$ & Females & & & 45 & 31 & & & & UNICEF & $\begin{array}{l}2004- \\
2016\end{array}$ \\
\hline
\end{tabular}


Table 3 Distribution of select NCD risk factors among adolescents by global region (Continued)

\begin{tabular}{|c|c|c|c|c|c|c|c|c|c|c|c|}
\hline \multicolumn{2}{|c|}{ Risk Factor Indicators } & \multirow[t]{2}{*}{ Sex } & \multicolumn{7}{|l|}{ Regions $^{1}$} & \multirow{2}{*}{$\begin{array}{l}\text { Data } \\
\text { Source }\end{array}$} & \multirow{2}{*}{$\begin{array}{l}\text { Year of } \\
\text { Data } \\
\text { Collection }\end{array}$} \\
\hline & & & Americas & Europe & $\begin{array}{l}\text { Eastern } \\
\text { and } \\
\text { Southern } \\
\text { Africa }\end{array}$ & $\begin{array}{l}\text { Western } \\
\text { and } \\
\text { Central } \\
\text { Africa }\end{array}$ & $\begin{array}{l}\text { Middle } \\
\text { East \& } \\
\text { North } \\
\text { Africa }\end{array}$ & Pacific & $\begin{array}{l}\text { South } \\
\text { Asia }\end{array}$ & & \\
\hline $\begin{array}{l}\text { Income } \\
\text { Inequality }\end{array}$ & GNI per capita (U.S.\$) [38] & & 8200 & 22,651 & $1454^{* *}$ & & 7246 & 10,170 & 1743 & $\begin{array}{l}\text { World } \\
\text { Bank }\end{array}$ & 2017 \\
\hline Urbanization & $\begin{array}{l}\% \text { of total population } \\
\text { urbanized [27] }\end{array}$ & & 80 & 64 & 31 & 45 & 63 & 57 & 33 & UNICEF & 2016 \\
\hline
\end{tabular}

${ }^{1}$ Regions are based on a combination of the seven UNICEF regions (Southern and Eastern Africa, West and Central Africa, Caribbean, Europe \& CIS, North Africa, Pacific, South Asia and Southern Africa) and six WHO regions (Africa, Americas, Europe, Mediterranean, South-East Asia, and Western Pacific) and when necessary data from the World Bank regions (East Asia \& Pacific, Europe \& Central Asia, Latin America \& Caribbean, Middle East \& North Africa, Sub-Saharan Africa, South Asia). The amalgamation of regions includes: the Americas (Caribbean UNICEF region, Americas WHO region, Latin America and Caribbean World Bank region), Europe region (Europe \& CIS UNICEF region, Europe WHO region and Europe \& Central Asia World Bank region), Middle East and North Africa region (Middle East and North Africa UNICEF region, Eastern Mediterranean Region WHO and Middle East \& North Africa World Bank region), Pacific region (Western Pacific WHO region, Pacific UNICEF region and East Asia \& Pacific World Bank region), Southern and Eastern Africa (Southern and Eastern Africa region UNICEF), Western and Central Africa region (UNICEF) and South Asia region (South Asia UNICEF region, South East Asia WHO region and South Asia World Bank region)

* = Regional estimates are only available for the WHO African region

** = Regional estimates are only available for World Bank Sub Saharan Africa region

$+=$ Regional estimates were calculated by weighting country estimates with population data from the respective year (e.g. 2010) using the United Nations

population prospects database and incorporating those into a combined estimate for the region

- = Regional data unavailable

pollution. These top factors were not different between sexes. These important risk factors even remained as the top contributors of later adulthood (50-69 years) disease-related disability and death (Additional file 1).

\section{Multivariable determinants of NCDs among adolescents and adults}

In our analysis of correlates of NCD DALYS among adolescents, at the distal level, macro factors such as indicators of conflict and humanitarian crises (e.g. refugee populations, $p<0.0001$ ), and state governance (effectiveness $[p<0.0001]$, corruption $[p<0.0001])$ were independently associated with higher NCD burden (Table 4). Across a range of statistically significant national structural factors at the intermediate I level, urbanization ( $22=14 \%, p<0.0001)$, GDP per capita ( $2=11 \%, p<$ $0.0001)$, and total health expenditure per capita $(\mathrm{R} 2=$ $12 \%, p<0.0001)$ were more strongly related to the outcome. At the intermediate II level, other indicators of household socioeconomic status, youth literacy and fertility rates, proxies of women's empowerment, income inequities, and availability of human resources for health were all statistically significant correlates of adolescent NCDs. In multivariable analyses, a final joint model explained about $62 \%$ of the variance in adolescent NCD DALYs worldwide. This included a series of distal and intermediate factors such as the total refugee populations in a country, effective state governance, urbanization, access to electricity, population density, total health expenditure per capita, adolescent fertility, and a proxy of female gender empowerment (secondary school gender parity index) (Table 4).

Our model of correlates of NCD DALYs among adult populations is displayed in Table 5. Distal level macro factors associated with the burden of NCDs among adults in bivariate analyses were government effectiveness $[p=0.0003]$ and corruption $[p=0.0119])$. At the intermediate I level, access to electricity ( $2=5 \%, p<$ $0.0001)$, mobile cellular subscriptions ( $2=15 \%, p<$ $0.0001)$, GDP per capita ( $2=9 \%, p<0.0001)$, and total health expenditure per capita ( $\mathrm{R} 2=8 \%, p<0.0001$ ) were strongly related to NCD burden among adults. The final adjusted model for adult populations included the distal and intermediate factors of corruption in state governance, urbanization, access to electricity, GDP per capita, secondary enrolment ratio, physician density per 1000 population as well as proxies for female gender empowerment (secondary and tertiary school gender parity indexes) (Table 5). It should be noted, however, that this adult model only explained about $31 \%$ of the variance in adulthood NCD suggesting that many other proximal factors may be at play (e.g. lifestyle - smoking, drinking, drug use, diet) which were not captured in our models. The higher \% variance explained in the adolescent model vs adult model suggests NCDs in adolescence are particularly vulnerable to broader macro and societal factors that are evaluated in this analysis.

\section{National Policies, Laws and Legislations for NCD prevention}

The availability of policies and laws targeting NCD-related lifestyle and behavioral risk factors among adolescents varied substantially across regions (Additional file 1). Across countries in the African region, the majority perform well with available national policies and strategies for sexual/ reproductive health/family planning (95\%), violence (85\%) and mental health (82\%), nutritional interventions (80\%), alcohol use prevention (79\%), tobacco control activities (79\%), and injury prevention (71\%). Similar patterns are 
Table 4 Hierarchical bivariate and multivariable determinants of NCD DALYs among adolescents in 194 countries, 2015

\section{Domain/Indicator \\ Level 3 Model- Distal: Macro Structural Factors ${ }^{\text {a }}$ \\ Conflict}

Battle related deaths (total; log)

Internally displaced persons (total; log)

Refugee populations by country of asylum (total; log)

\section{Governance}

Political stability/absence of terrorism

Government effectiveness ${ }^{1}$

Corruption $^{1}$

\section{Environment}

Frequency of natural disasters (total; log)

Cost damage of natural disasters (USD; log)

\section{Level 2 Model- Intermediate I: National Structural Factors ${ }^{\mathbf{b}}$} Infrastructure and Urbanization

Urbanization (\% of population; log)

Access to electricity (\% population; cubed)

\section{Population Density}

Population density (people per $\mathrm{m}^{2}$ land; log)

\section{Telecommunications Access}

Mobile cellular subscriptions (per 100 people; log)

Internet users (per 100 people; log)

\section{National Wealth}

GDP per capita, 5 year lag (USD; log) ${ }^{3}$

\section{Health Spending}

Government expenditure on health, 2 year lag (\% of total health expenditure; log)

Total health expenditure per capita, 2 year lag (PPP, NCU per USD; $\log )^{3}$
Outcome: DALYs attributed to NCDs among adolescents (rate, 2015)

\begin{tabular}{|c|c|c|c|}
\hline \multicolumn{3}{|c|}{ Bivariate } & \multirow{2}{*}{$\begin{array}{l}\text { Multivariable } \\
\text { B estimate } \\
\text { (Standard error) } \\
\text { P-value }\end{array}$} \\
\hline $\begin{array}{l}\text { R- } \\
\text { Square }\end{array}$ & $\begin{array}{l}\text { B estimate } \\
\text { (Standard error) } \\
P \text {-value }\end{array}$ & StB & \\
\hline
\end{tabular}

\section{Source \& Year}

\begin{tabular}{|c|c|c|c|c|}
\hline World Bank, 2013 & $1 \%$ & $\begin{array}{l}-23.5(16.92) \\
0.1673\end{array}$ & $-\overline{0}$ & - \\
\hline World Bank, 2013 & $1 \%$ & $\begin{array}{l}-21.8(24.92) \\
0.3838\end{array}$ & $\begin{array}{l}- \\
0.07\end{array}$ & - \\
\hline World Bank, 2014 & $16 \%$ & $\begin{array}{l}-58.3(10.31) \\
<.0001\end{array}$ & $-\overline{0.39}$ & $\begin{array}{l}-19.8(8.13) \\
0.0156\end{array}$ \\
\hline World Bank, 2013 & $0 \%$ & $\begin{array}{l}-11.3(60.98) \\
0.8534\end{array}$ & -0.01 & - \\
\hline World Bank, 2013 & $11 \%$ & $\begin{array}{l}-264.4(56.03) \\
<.0001\end{array}$ & -0.33 & $\begin{array}{l}-442.4(44.27) \\
<.0001\end{array}$ \\
\hline \multirow[t]{3}{*}{ World Bank, 2013} & $10 \%$ & $\begin{array}{l}-245.9(56.74) \\
<.0001\end{array}$ & -0.31 & - \\
\hline & $0 \%$ & $\begin{array}{l}-72.2(84.02) \\
0.3914\end{array}$ & -0.07 & - \\
\hline & $2 \%$ & $\begin{array}{l}-11.7(6.91) \\
0.0924\end{array}$ & -0.13 & - \\
\hline
\end{tabular}

Distal Model R2: $38 \%$

\begin{tabular}{|c|c|c|c|c|}
\hline UNICEF, 2016 & $14 \%$ & $\begin{array}{l}-597.0(111.74) \\
<.0001\end{array}$ & -0.37 & $\begin{array}{l}-261.0(120.39) \\
0.0316\end{array}$ \\
\hline World Bank & $9 \%$ & $\begin{array}{l}-0.00057(0.00014) \\
<.0001\end{array}$ & -0.30 & $\begin{array}{l}-0.0005(0.00016) \\
0.0014\end{array}$ \\
\hline World Bank & $2 \%$ & $\begin{array}{l}-91.2(43.15) \\
0.036\end{array}$ & -0.16 & $\begin{array}{l}-52.8(33.41) \\
0.1162\end{array}$ \\
\hline World Bank, 2014 & $3 \%$ & $\begin{array}{l}-266.1(114.17) \\
0.0209\end{array}$ & -0.17 & - \\
\hline World Bank, 2014 & $6 \%$ & $\begin{array}{l}-147.7(43.14) \\
0.0008\end{array}$ & -0.25 & - \\
\hline World Bank, 2014 & $11 \%$ & $\begin{array}{l}-172.9(37.70) \\
<.0001\end{array}$ & -0.33 & - \\
\hline WHO, 2013 & $1 \%$ & $\begin{array}{l}-165.0(151.16) \\
0.2766\end{array}$ & -0.08 & - \\
\hline \multirow[t]{2}{*}{ WHO, 2013} & $12 \%$ & $\begin{array}{l}-169.7(34.83) \\
<.0001\end{array}$ & -0.34 & $\begin{array}{l}-111.3(62.56) \\
0.0771\end{array}$ \\
\hline & & & & $\begin{array}{l}\text { Intermediate I } \\
\text { Model R2: } \\
47 \%\end{array}$ \\
\hline
\end{tabular}


Table 4 Hierarchical bivariate and multivariable determinants of NCD DALYs among adolescents in 194 countries, 2015 (Continued)

\section{Domain/Indicator}

Outcome: DALYs attributed to NCDs among adolescents (rate, 2015)

\begin{tabular}{|c|c|c|c|c|c|}
\hline \multirow{3}{*}{\multicolumn{2}{|c|}{$\begin{array}{l} \\
\text { Level 3- Intermediate II: Community, Household \& Individual Factors }{ }^{c}\end{array}$}} & \multirow{2}{*}{\multicolumn{3}{|c|}{ Bivariate }} & \multirow{3}{*}{ Multivariable } \\
\hline & & & & & \\
\hline & & \multicolumn{3}{|c|}{ Level 3- Intermediate II: Community, Household \& Individual Factors c } & \\
\hline \multicolumn{6}{|l|}{ Socioeconomic Status (Education and Employment) } \\
\hline Adult literacy rate ( $\%$ of adults ages $15+$ years; cubed) & World Bank & $1 \%$ & $\begin{array}{l}-0.00024(0.00022) \\
0.2767\end{array}$ & -0.10 & \\
\hline Primary school enrolment ratio (gross \%; log) & $\begin{array}{l}\text { World Bank, } \\
\text { 2003-2014 }\end{array}$ & $1 \%$ & $\begin{array}{l}719.0(499.43) \\
0.1519\end{array}$ & 0.11 & \\
\hline Secondary school enrolment ratio (gross \%; log) & World Bank, 2013 & $7 \%$ & $\begin{array}{l}-496.9(144.18) \\
0.0007\end{array}$ & -0.27 & \\
\hline Employment to population ratio (\% of adults $15+$ years; log) & World Bank & $4 \%$ & $\begin{array}{l}724.5(282.59) \\
0.0112\end{array}$ & 0.19 & \\
\hline \multicolumn{6}{|l|}{ Youth Empowerment } \\
\hline Youth literacy rate (\% total 15-24 year olds; squared) & World Bank, 2014 & $3 \%$ & $\begin{array}{l}-0.1(0.03) \\
0.0718\end{array}$ & -0.16 & - \\
\hline Female youth literacy rate (\% 15-24 year olds; squared) & World Bank, 2014 & $2 \%$ & $\begin{array}{l}-0.04(0.03) \\
0.1169\end{array}$ & $-\overline{0} \cdot 14$ & - \\
\hline Youth unemployment rate (\% total 15-24 year olds; log) & World Bank, 2013 & $1 \%$ & $\begin{array}{l}-95.3(76.09) \\
0.212\end{array}$ & $\overline{0}-10$ & - \\
\hline $\begin{array}{l}\text { Adolescent fertility rate (births per } 1000 \text { females aged 15-19 } \\
\text { years; log) }\end{array}$ & $\begin{array}{l}\text { World Bank, } \\
\text { 2009-2014 }\end{array}$ & $12 \%$ & $\begin{array}{l}256.0(52.22) \\
<.0001\end{array}$ & 0.34 & $\begin{array}{l}224.4(66.17) \\
0.0009\end{array}$ \\
\hline \multicolumn{6}{|l|}{ Women Empowerment and Gender Equity } \\
\hline Total fertility rate (births per woman; log) & World Bank, 2013 & $7 \%$ & $\begin{array}{l}454.5(122.04) \\
0.0003\end{array}$ & 0.27 & - \\
\hline $\begin{array}{l}\text { Adult female literacy rate (\% females } 15+\text { years who can read } \\
\text { and write; cubed) }\end{array}$ & World Bank & $0.5 \%$ & $\begin{array}{l}-0.016(0.021) \\
0.4481\end{array}$ & -0.07 & - \\
\hline $\begin{array}{l}\text { Women in parliament (\% of parliamentary seats held by women; } \\
\text { log) }\end{array}$ & World Bank, 2014 & $4 \%$ & $\begin{array}{l}-92.3(35.97) \\
0.0111\end{array}$ & -0.19 & - \\
\hline $\begin{array}{l}\text { Secondary school gender parity index (ratio of girls to boys in } \\
\text { secondary education; log) }\end{array}$ & World Bank, 2013 & $3 \%$ & $\begin{array}{l}-877.5(416.20) \\
0.0366\end{array}$ & -0.17 & $\begin{array}{l}-700.4(341.58) \\
0.0422\end{array}$ \\
\hline $\begin{array}{l}\text { Tertiary school gender parity index (ratio of girls to boys in } \\
\text { tertiary education; log) }\end{array}$ & World Bank, 2013 & $6 \%$ & $\begin{array}{l}-206.4(66.82) \\
0.0024\end{array}$ & $\overline{0} .25$ & - \\
\hline \multicolumn{6}{|l|}{ Income Equity } \\
\hline GINI index $(\log )^{2}$ & World Bank, 2012 & $12 \%$ & $\begin{array}{l}1072.6(269.17) \\
0.0001\end{array}$ & 0.35 & - \\
\hline \multicolumn{6}{|l|}{ Access to Health Services and Commodities } \\
\hline Out of pocket expenditure as \% of total health expenditure (log) & WHO, 2013 & $0.06 \%$ & $\begin{array}{l}-23.7(75.81) \\
0.7544\end{array}$ & -0.02 & - \\
\hline \multirow[t]{2}{*}{ Physician density per 1000 population (log) } & WHO, 2003-2013 & $10 \%$ & $\begin{array}{l}-177.1(47.11) \\
0.0003\end{array}$ & $\begin{array}{l}- \\
0.32\end{array}$ & - \\
\hline & & & & & $\begin{array}{l}\text { Intermediate II } \\
\text { Model R2: } \\
62 \%\end{array}$ \\
\hline
\end{tabular}

Note: variables significant at $p<0.20$ in bivariate analysis were entered into elastic net linear regression models; ${ }^{1}$ Government effectiveness and corruption were strongly collinear $(>80 \%)$ and thus only the former was entered into multivariable modeling; ${ }^{2}$ Due to small sample size ( $n=117$ countries), GINI index not considered in multivariable analysis; ${ }^{1}$ GDP per capita and health expenditure per capita were strongly collinear (> $\left.80 \%\right)$ and thus only the latter was entered into multivariable modeling

${ }^{\text {a }}$ Level 3 multivariable model includes all statistically significant $(p<0.15)$ distal variables as listed

b Level 2 multivariable model includes level 3 model+ all statistically significant $(p<0.15)$ intermediate I variables as listed

c Level 1 multivariable model includes level 2 model+ all statistically significant $(p<0.15)$ intermediate II variables as list

observed in the South-East Asian region. Both regions are lacking in policies where laws and regulations allow minors to seek contraceptive services and emergency contraception without parental/caregiver consent and seek harm reduction interventions for injectable drug use. Policies which exempt adolescents aged 15-19 years from 
Table 5 Hierarchical bivariate and multivariable determinants of NCD DALYs among adults in 194 countries, 2015

\section{Domain/Indicator \\ Level 3 Model- Distal: Macro Structural Factors ${ }^{a}$ \\ Conflict}

Outcome: DALYs attributed to NCDs among adults (rate, 2015)

\begin{tabular}{|c|c|c|c|}
\hline \multicolumn{3}{|c|}{ Bivariate } & \multirow{2}{*}{$\begin{array}{l}\text { Multivariable } \\
\text { B estimate } \\
\text { (Standard error) } \\
P \text {-value }\end{array}$} \\
\hline $\begin{array}{l}\text { R- } \\
\text { Square }\end{array}$ & $\begin{array}{l}\text { B estimate } \\
\text { (Standard error) } \\
\text { P-value }\end{array}$ & StB & \\
\hline
\end{tabular}

Battle related deaths (total; log)

Internally displaced persons (total; log)

Refugee populations by country of asylum (total; log)

\section{Governance}

Political stability/ absence of terrorism

Government effectiveness ${ }^{1}$

Corruption $^{1}$

\section{Environment}

Frequency of natural disasters (total; log)

Cost damage of natural disasters (USD; log)

\section{Level 2 Model- Intermediate I: National Structural Factors ${ }^{\text {b }}$}

\section{Infrastructure and Urbanization}

Urbanization (\% of population; log)

Access to electricity (\% population; cubed)

\section{Population Density}

Population density (people per $\mathrm{m}^{2}$ land; log)

\section{Telecommunications Access}

Mobile cellular subscriptions (per 100 people; log)

Internet users (per 100 people; log)

\section{National Wealth}

GDP per capita, 5 year lag (USD; log) ${ }^{2}$

\section{Health Spending}

Government expenditure on health, 2 year lag (\% of total health WHO, 2013 expenditure; log)

Total health expenditure per capita, 2 year lag (PPP, NCU per USD; $\log )^{2}$

\begin{tabular}{|c|c|c|c|}
\hline \multicolumn{4}{|l|}{ Source \& Year } \\
\hline World Bank, 2013 & $9 \%$ & $\begin{array}{l}492.95(242.94) \\
0.0487\end{array}$ & 0.17 \\
\hline World Bank, 2013 & $0 \%$ & $\begin{array}{l}31.75(218.61) \\
0.8847\end{array}$ & 0.01 \\
\hline World Bank, 2014 & $3 \%$ & $\begin{array}{l}-262.897(127.60) \\
0.0410\end{array}$ & -0.13 \\
\hline World Bank, 2013 & $2 \%$ & $\begin{array}{l}-960.160(512.51) \\
0.0673\end{array}$ & -0.13 \\
\hline World Bank, 2013 & $7 \%$ & $\begin{array}{l}-1831.88(498.67) \\
0.0003\end{array}$ & -0.26 \\
\hline \multirow[t]{3}{*}{ World Bank, 2013} & $4 \%$ & $\begin{array}{l}-1288.79(507.14) \\
0.0119\end{array}$ & -0.18 \\
\hline & $0.3 \%$ & $\begin{array}{l}-487.76(751.42) \\
0.5172\end{array}$ & -0.05 \\
\hline & $0.8 \%$ & $\begin{array}{l}-257.35(403.53) \\
0.5266\end{array}$ & -0.09 \\
\hline
\end{tabular}

Distal Model R2: $4 \%$

\begin{tabular}{|c|c|c|c|c|}
\hline UNICEF, 2016 & $6 \%$ & $\begin{array}{l}-3405.14(1004.45) \\
.0009\end{array}$ & -0.24 & $\begin{array}{l}-2668.44(1064.88) \\
0.0131\end{array}$ \\
\hline World Bank & $5 \%$ & $\begin{array}{l}-0.0037(0.0012) \\
<.0001\end{array}$ & -0.22 & $\begin{array}{l}0.0025(0.0015) \\
0.1040\end{array}$ \\
\hline World Bank & $0.8 \%$ & $\begin{array}{l}-445.2(378.10) \\
0.2406\end{array}$ & -0.09 & - \\
\hline World Bank, 2014 & $15 \%$ & $\begin{array}{l}-5158.49(919.96) \\
<.0001\end{array}$ & -0.38 & - \\
\hline World Bank, 2014 & $5 \%$ & $\begin{array}{l}-1098.425(376.30) \\
0.0040\end{array}$ & -0.17 & - \\
\hline World Bank, 2014 & $9 \%$ & $\begin{array}{l}-1168.48(271.40) \\
<.0001\end{array}$ & -0.25 & $\begin{array}{l}-1515.97(586.38) \\
0.0105\end{array}$ \\
\hline WHO, 2013 & $4 \%$ & $\begin{array}{l}-2627.68(1032.80) \\
0.0118\end{array}$ & -0.15 & - \\
\hline \multirow[t]{2}{*}{ WHO, 2013} & $8 \%$ & $\begin{array}{l}-999.81(250.55) \\
<.0001\end{array}$ & -0.23 & - \\
\hline & & & & $\begin{array}{l}\text { Intermediate I } \\
\text { Model R2: } \\
14 \%\end{array}$ \\
\hline
\end{tabular}


Table 5 Hierarchical bivariate and multivariable determinants of NCD DALYs among adults in 194 countries, 2015 (Continued)

\begin{tabular}{|c|c|c|c|c|c|}
\hline \multirow{2}{*}{\multicolumn{2}{|c|}{ Domain/Indicator }} & \multicolumn{4}{|c|}{$\begin{array}{l}\text { Outcome: DALYs attributed to NCDs among adults } \\
\text { (rate, 2015) }\end{array}$} \\
\hline & & \multicolumn{3}{|c|}{ Bivariate } & \multirow[t]{2}{*}{ Multivariable } \\
\hline \multicolumn{5}{|l|}{ Level 3- Intermediate II: Community, Household \& Individual Factors ${ }^{c}$} & \\
\hline \multicolumn{6}{|l|}{ Socioeconomic Status (Education and Employment) } \\
\hline Adult literacy rate (\% of adults ages $15+$ years; cubed) & World Bank & $0.7 \%$ & $\begin{array}{l}-0.0015(0.0016) \\
0.3635\end{array}$ & -0.07 & - \\
\hline Primary school enrolment ratio (gross \%; log) & $\begin{array}{l}\text { World Bank, } \\
\text { 2003-2014 }\end{array}$ & $0.1 \%$ & $\begin{array}{l}1311.79(3489.26) \\
0.7074\end{array}$ & 0.02 & - \\
\hline Secondary school enrolment ratio (gross \%; log) & UNICEF, 2013 & $1 \%$ & $\begin{array}{l}-1465.82(990.54) \\
0.1410\end{array}$ & -0.09 & $\begin{array}{l}4166.21(2642.13) \\
0.1182\end{array}$ \\
\hline Employment to population ratio (\% of adults $15+$ years; log) & World Bank & $0.2 \%$ & $\begin{array}{l}1325.91(2539.92) \\
0.6023\end{array}$ & 0.04 & \\
\hline \multicolumn{6}{|l|}{ Youth Empowerment } \\
\hline Youth literacy rate (\% total $15-24$ year olds; squared $)^{4}$ & World Bank, 2014 & $2 \%$ & $\begin{array}{l}-0.3266(0.2154) \\
0.1320\end{array}$ & -0.11 & - \\
\hline Female youth literacy rate (\% 15-24 year olds; squared $)^{4}$ & World Bank, 2014 & $1 \%$ & $\begin{array}{l}-0.2504(0.1997) \\
0.2125\end{array}$ & -0.09 & - \\
\hline Youth unemployment rate (\% total 15-24 year olds; log) & World Bank, 2013 & $0 \%$ & $\begin{array}{l}1.5364(672.15) \\
0.9982\end{array}$ & 0.0002 & - \\
\hline $\begin{array}{l}\text { Adolescent fertility rate (births per } 1000 \text { females aged 15-19 } \\
\text { years; log) }\end{array}$ & $\begin{array}{l}\text { World Bank, } \\
\text { 2009-2014 }\end{array}$ & $0 \%$ & $\begin{array}{l}-81.08(485.13) \\
0.8675\end{array}$ & -0.012 & - \\
\hline \multicolumn{6}{|l|}{ Women Empowerment and Gender Equity } \\
\hline Total fertility rate (births per woman; log) & World Bank, 2013 & $0.7 \%$ & $\begin{array}{l}1223.67(1106.03) \\
0.2700\end{array}$ & 0.08 & - \\
\hline $\begin{array}{l}\text { Adult female literacy rate (\% females } 15+\text { years who can read } \\
\text { and write; cubed) }\end{array}$ & World Bank & $0.3 \%$ & $\begin{array}{l}-0.0010(0.0015) \\
0.5232\end{array}$ & -0.05 & - \\
\hline $\begin{array}{l}\text { Women in parliament (\% of parliamentary seats held by } \\
\text { women; log) }\end{array}$ & World Bank, 2014 & $2 \%$ & $\begin{array}{l}-1270.29(714.36) \\
0.0771\end{array}$ & -0.13 & - \\
\hline $\begin{array}{l}\text { Secondary school gender parity index (ratio of girls to boys in } \\
\text { secondary education; log) }\end{array}$ & World Bank, 2013 & $2 \%$ & $\begin{array}{l}-5512.67(2829.38) \\
0.0532\end{array}$ & -0.12 & $\begin{array}{l}-18,846.97(5432.46) \\
0.0008\end{array}$ \\
\hline $\begin{array}{l}\text { Tertiary school gender parity index (ratio of girls to boys in } \\
\text { tertiary education; log) }\end{array}$ & World Bank, 2013 & $2 \%$ & $\begin{array}{l}-1268.27(834.61) \\
0.1308\end{array}$ & -0.09 & $\begin{array}{l}3176.62(1776.29) \\
0.0770\end{array}$ \\
\hline \multicolumn{6}{|l|}{ Income Equity } \\
\hline GINI index (log) & World Bank, 2012 & $0 \%$ & $\begin{array}{l}312.54(2260.12) \\
0.8903\end{array}$ & 0.01 & - \\
\hline \multicolumn{6}{|l|}{ Access to Health Services and Commodities } \\
\hline $\begin{array}{l}\text { Out of pocket expenditure as \% of total health expenditure } \\
\text { (log) }\end{array}$ & WHO, 2013 & $0.5 \%$ & $\begin{array}{l}-513.4(524.81) \\
0.3292\end{array}$ & -0.07 & - \\
\hline \multirow[t]{2}{*}{ Physician density per 1000 population (log) } & WHO, 2003-2013 & $3 \%$ & $\begin{array}{l}-715.62(366.23) \\
0.0529\end{array}$ & -0.15 & $\begin{array}{l}1786.88(844.88) \\
0.0371\end{array}$ \\
\hline & & & & & $\begin{array}{l}\text { Intermediate II } \\
\text { Model R2: } \\
31 \%\end{array}$ \\
\hline
\end{tabular}

Note: variables significant at $p<0.20$ in bivariate analysis were entered into elastic net linear regression models; ${ }^{1}$ Political stability and Corruption were strongly collinear $(>80 \%)$ and thus only the later was entered into multivariable modeling; ${ }^{2}$ Health expenditure per capita and GDP per capita were strongly collinear (> $80 \%)$ and thus only the latter was entered into multivariable modeling; ${ }^{3}$ Due to small sample size ( $n=117$ countries), GINI index not considered in multivariable analysis; ${ }^{4}$ Female Youth Literacy Rate and Youth Literacy Rate Both were strongly collinear $(>80 \%)$ and thus only the latter was entered into multivariable modeling

a Level 3 multivariable model includes all statistically significant $(p<0.15)$ distal variables as listed

${ }^{\mathrm{b}}$ Level 2 multivariable model includes level 3 model+ all statistically significant $(p<0.15)$ intermediate I variables as listed

c Level 1 multivariable model includes level 2 model+ all statistically significant $(p<0.15)$ intermediate II variables as list 

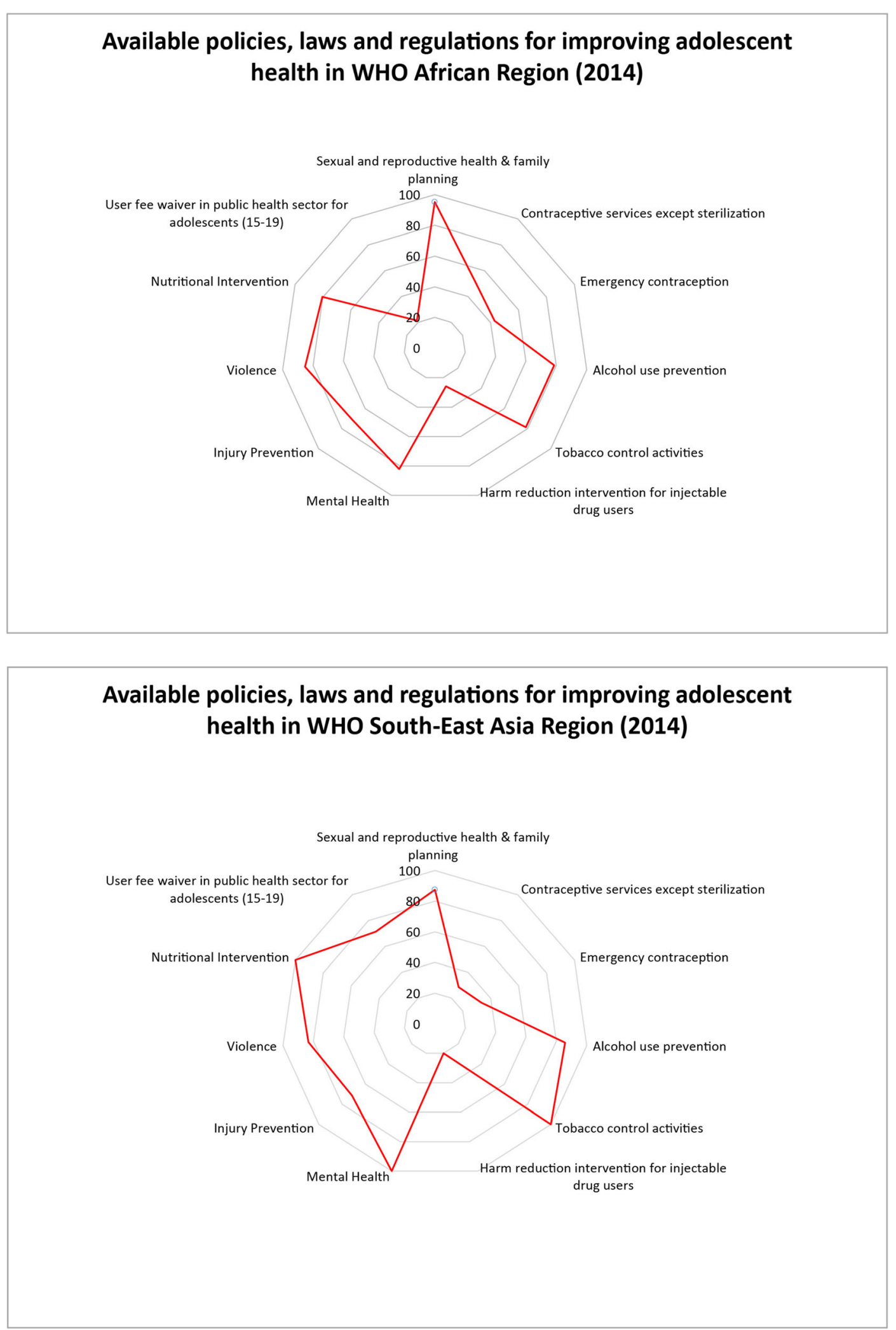

Fig. 2 Policies, laws and regulations for adolescent NCD prevention in Africa and South East Asia 
user fees in the public sector are more common in SouthEast Asia (71\% of countries) and are lacking in Africa (21\%) (Fig. 2). Across other regions (Additional file 1), the Western Pacific region specifically lacked in policies on mental health and nutritional interventions. In the Eastern Mediterranean, less than $45 \%$ of countries had adolescentspecific policies on alcohol use and injury/violence prevention. Among Eastern Mediterranean region countries with available data, none had available national laws and regulations permitting adolescents to seek contraceptive services or emergency contraception, and for harm reduction interventions for illicit drug users. In fact, these were lacking in all regions. However, not all countries in these regions participated in the surveys, and sample sizes vary by region and within region by policy type.

\section{Evidence-based interventions and delivery platforms for NCD prevention}

Evidence-based interventions for adolescent populations that specifically target their modifiable risk factors for NCDs in adolescent and later life, can be broadly categorized into those delivered through community-based, school-based, peer-based, and family-based platforms. Varying in their degrees of effectiveness and implementation in high-income countries (HICs) versus low-andmiddle income countries (LMICs), these interventions have been used to address NCD risk factors and promote protective factors among adolescents. We detail effective interventions for NCD prevention and reduction among adolescents [11, 42-108], and provide a projectspecific overview in the appendix (Additional file 1).

Implementing evidence-based initiatives as part of a multi-level, multi-component and inter-sectoral approach can enhance their effectiveness by providing holistic and sustainable solutions [12]. For instance, interventions incorporating policy measures, environmental changes for promoting physical activity, and education on healthy diet and physical education simultaneously have been more effective in addressing obesity among adolescents compared to individual interventions [109]. Another example is cash incentive programs, which seek to improve school retention, while addressing issues of poverty, by providing payment incentives to students and their parents. These programs have been effective in improving educational attainment and decreased aggression, crime rates, alcohol and tobacco use, unwanted pregnancies, and mental health symptoms and disorders. Moreover, these have achieved long-term impact post-intervention, that last between 1 and 15 years $[105,110-112]$. Lastly, mass media and social marketing interventions that target adolescents, their families, and broader communities, have been effective in altering social norms and have served as a platform for grassroots movements by empowering adolescents and broader communities [12, 113]. Recent advances in digital technology such as widespread availability and use of mobile phones, social media and online games may also serve as valuable intervention platforms for adolescents. Overall, efforts aimed at improving NCDs among adolescents and in later life should consider taking on holistic solutions that seek to build capacity within individual sectors while enhancing coordination across sectors.

\section{Discussion}

We conducted a comprehensive review of NCD burden and determinants among adolescents, and also derived an evidence-based conceptual framework for tracking pathways to NCD development. We noted that NCDs are a prevailing public health concern among adolescents globally, among which, mental health conditions including depression and conduct disorders are leading disorders among both males and females. Proximal behavioral and lifestyle risk factors are important to disease burden among adolescents, and our analyses show that much of the burden of NCDs in adulthood are related to these modifiable factors that start in adolescence. Most notably, poor diets, smoking, alcohol use, drug use, and indicators of metabolic syndrome (high blood pressure, high cholesterol, diabetes) are the largest contributors to adulthood NCDs and the risks of each of these typically begin in adolescence. Our statistical model also demonstrates that these proximal factors are influenced by broader community and macro factors including social equality, youth empowerment, economic growth and health expenditure, infrastructure and state development, and good governance and security/stability. We also found that, globally, there is momentum towards implementing NCD specific policies/laws/legislations at the national level, but that there is a general lack of policies and laws allowing minors to seek contraceptive services and emergency contraception without consent, and to seek harm reduction interventions for injectable drug use. Additionally, mental health and nutrition related policies exist only variably across geographic regions.

Another consideration are the commercial determinants of health, which refer to consumption of commercial products such as processed food, tobacco, and alcohol, and the vested interests of corporations to encourage their use [114]. The commercial drivers of ill health are related to the lifestyle risk factors presented in our work as the tobacco, alcohol, and processed food and beverage industries influence consumption of products related to poor diets, smoking, and alcohol use, which in turn impact adolescent NCD burden $[6,115]$. The global food industry has been identified as the leading driver of NCD epidemics related to diet [116]. The commercial determinants of health fall under the larger 
macro, societal and political social determinants of NCDs in adolescents, and they modify the behaviours of individuals in meaningful ways.

Our conceptual framework allows for the visualization of the diverse and complex web of pathways that shape NCDs among young people and in later life. In addition, it identifies critical windows or time periods for intervention, as well as the types of interventions that are necessary to mitigate and address the current and future burden. This conceptual framework could also be used to guide and strengthen the monitoring and evaluation of NCDs among adolescents that is urgently needed. Research on NCDs among adolescents has substantially focused on their engagement in key risk and protective behaviours [5, 12]. However, this study emphasizes that a paradigm shift is needed in order to recognize the critical role of underlying structural and societal determinants of health on NCDs among adolescents.

Key global and regional trends among adolescents highlight the significant burden of NCDs with a particular emphasis on mental illnesses such as conduct and major depressive disorders. This burden persists despite many countries globally, including $82 \%$ of countries in the African region, reporting the introduction of mental health policies. These trends may indicate weak implementation of key policies, funding, and political will to address NCDs among young people in many LMICs.

Risk and protective factors for NCDs among adolescents, including tobacco smoking, use of alcohol, physical inactivity, unemployment, and overweight, vary widely by region and sex. These findings align with previous empirical research on the burden of disease, risk and health behaviours among adolescent populations [5]. It also emphasizes the need to reduce the current burden of NCDs among young people, and prevent the heavy adult mortality and morbidity burden associated with risk factors for NCDs acquired in early life [5]. The diverse trends and inequities highlight the need to develop regional- and country-specific policies and programs to target key contributors to the NCD burden according to need.

Macro and societal factors across the life-course play a critical role on the burden of current and future NCDs among young people. Basic security, humanitarian issues and effective governance represent significant underlying structural determinants that shape both intermediate and proximal factors. Furthermore, infrastructure (e.g., urbanization and access to electricity), access to resources, health spending, human resources for health, gender equity and youth empowerment represent key intermediary influences that contribute significantly to NCDs among adolescents. These findings challenge the dominant perspective that individual and lifestyle risk and protective factors represent the primary contributors to NCDs, and emphasize the importance of underlying macro-level determinants. Other studies have also noted that broader social and economic determinants of health are linked to mortality, morbidity, and risk and protective factors among adolescents [12]. A paradigm shift in the conceptualization of NCDs and risk factors is therefore critical to developing effective interventions and policies to prevent and mitigate the increasing burden among adolescents. Investments in and emphasis on using multi-level and cross-sectoral interventions and policies that address these diverse influences must be prioritized by countries globally to achieve improvements in NCDs among adolescents. For example, current and future efforts to reduce the risk factors associated with NCDs could be focused on developing primary health-care hubs at the lowest possible level of the health-care system with essential infrastructure and human resources [6]. Health services that include NCD management and screening in community health units in villages have reported benefits in Ethiopia, Malawi, Namibia, Rwanda, and Uganda [117, 118]. These initiatives have trained community health workers to deliver integrated programs for multiple conditions at the community level and to address the needs of women and children which has resulted in improved health outcomes [117, 118]. Another focal point in the efforts to combat NCDs is the synergistic effort to reduce health inequalities and improve the equity of government health expenditure by financing universal health coverage [119]. Rwanda has used funding from HIV programs to expand health insurance coverage for poor sections of the population to improve access to health services, including those for NCDs [119]. While there is limited information to differentiate between the initiatives focused on adolescent or adult populations specifically, our results are congruent with the current focus on reducing NCD rates by means of supporting the improvement of state governance and increasing health expenditure to decrease health, gender, and socioeconomic inequalities. Urbanization and improved country development are suggestive of overall economic development and can be signals of improved access to health services for the management and treatment of NCDs.

Our work found that policies/laws/legislations targeting NCD risk factors among adolescents generally appear to exist in many countries, but actual implementation and impact data on these NCD policies and laws is limited. Although the MNCAH policy indicator dashboard provides information on the availability of policies or laws on NCDs by region and country, it lacks detailed information on implementation and monitoring and evaluation for impact. There is a strong need for the latter in particular as it's needed to ensure and improve the quality and sustainability of programs, 
interventions, and policies targeting NCD risk factors. Tracking and holding countries accountable to their commitments for preventing NCDs is also critical, and can be initiated through preparing a core set of NCD monitoring indicators that can be used to evaluate and inform programs and policies that target NCD risk factors and health outcomes.

Our study is unique in that it used robust statistical modelling methods to explore the contributions of underlying distal, intermediate and proximal determinants in shaping the burden of NCDs among young people and adults. However, a few limitations should be noted. Firstly, our analysis was ecological (country-level) and thus results may be prone to the ecological fallacy. The sample size $(n=$ 194) was not large but our final models were sufficiently powered with the number of covariates included. Our crosssectional analysis could not infer temporality and associations do not necessarily suggest causality. Several important indicators (e.g. information on peer, family, and community social support systems, interpersonal relationships) and nutrient deficiency (e.g. anemia) and dietary intake did not have adequate available data for analysis and thus their effect should not be understated. For instance, while IHME databases have indicators on prevalence/incidence for dietary iron deficiency and hemoglobinopathies and hemolytic anemias for adolescents, estimates for many LMICs is missing or incomplete, and therefore we could not use this data in our analysis. Moreover, data used in this study are from publicly available sources such as the Global Health Observatory Data Repository and the World Bank database, and thus analyses and inference are limited to the quality of these data repositories. These sources often rely on survey datasets and other administrative sources that have known challenges in LMICs [120], and estimates may be modeled or direct estimates. However these estimates are amongst the best available globally and inferences will be meaningful, nonetheless must be interpreted with caution. The WHO MNCAH policy dashboard collects data only on existence of policies and thus the analysis in this study may not be representative of the country's actual policy implementation impact.

Efforts to prevent the burden of NCDs among adolescents and in later adult life represents an area that necessitates further research, investment and intervention. Key stakeholders, including State and non-State actors, working to improve adolescent health should address under-researched and under-funded areas that represent critical determinants of NCD burden and illness in many LMICs [106]. Evidence from this study can be used to re-frame the current situation of NCDs in adolescents, highlighting their pathways/determinants and existence (or lack thereof) of interventions/strategies for countering the diseases. Our findings can be used by UN bodies, government/policy-makers, development partners, and academia to target areas of concern for intervention and for identifying future research priorities. The critical role of structural and societal determinants on NCDs in this population must be recognized, including national governance and youth empowerment. Efforts to address these underlying influences require interventions and policies that span multiple sectors and determinants.

\section{Conclusions}

The findings of this study demonstrate the importance of adopting a more holistic approach to the prevention and reduction of NCD burden among adolescents, globally. The development and implementation of this approach requires a multilevel design that applies a life course perspective and addresses determinants across individual, community, national and societal levels. In addition, cross-sectoral collaboration is critical to ensuring effective development and implementation of policies to prevent NCDs among young people.

\section{Supplementary Information}

The online version contains supplementary material available at https://doi. org/10.1186/s12889-020-09988-5.

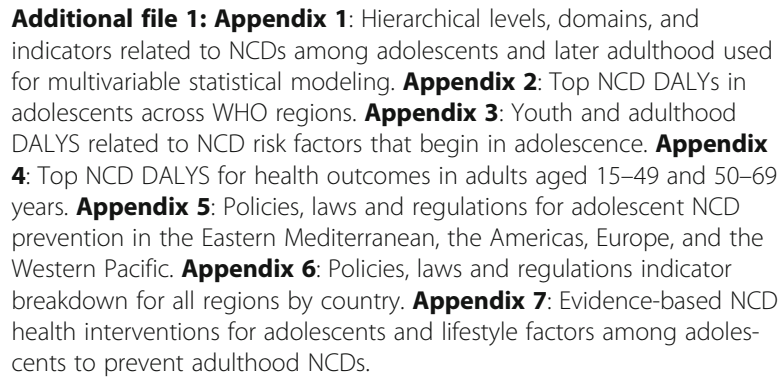

\section{Abbreviations}

DALY: Disability-adjusted life years; GBD: Global burden of disease; IHME: Institute for health metrics and evaluation; LMIC: Low and middle income country; MNCAH: Maternal, newborn, child, and adolescent health; NCD: Non-communicable disease

\section{Acknowledgements}

We would like to acknowledge the support of Hana Tasic, research assistant in the Centre for Global Child Health, Hospital for Sick Children, for her editorial review of this paper.

\footnotetext{
Authors' contributions

NA conceptualized the study, designed the analytic plan, conducted statistical analysis, drafted sections of the manuscript, critically reviewed the manuscript and approved the final manuscript as submitted. RC conducted the policy analysis, ZB drafted study tables, BS conducted statistical analysis and SAG drafted the section on evidence-based interventions. SM, JW, RC, $B S, Z B$ and SAG drafted sections of manuscript, conducted data extraction, critically reviewed the manuscript and approved the final manuscript as submitted. AV supported development of the conceptual framework, critically reviewed the manuscript and approved the final manuscript as submitted. $\mathrm{DH}$ and NS reviewed and revised the study protocol, critically reviewed the manuscript and approved the final manuscript as submitted. ZAB provided overall technical oversight, reviewed and revised the study protocol, critically reviewed the manuscript and approved the final manuscript as submitted. All authors read and approved the final manuscript.
} 


\section{Funding}

This study was funded by UNICEF and the Centre for Global Child Health at the Hospital for Sick Children. The funder commissioned the review, but had no role in the research process, methods or inferences from the analyses.

\section{Availability of data and materials}

The datasets analyzed during the current study are available in the Global Health Observatory Data Repository, https://www.who.int/gho/en/, the State of the World's Children global statistics database, https://data.unicef.org/ resources/state-worlds-children-2017-statistical-tables/, the World Bank database, https://data.worldbank.org/, the United Nations Statistics Division,http://www.un.org/en/development/desa/publications/worldpopulation-prospects-2015-revision.html, and the 2015Global Burden of Disease (GBD) study housed at the Institute for Health Metrics and Evaluation (IHME), http://www.healthdata.org/gbd.

\section{Ethics approval and consent to participate}

Not applicable.

\section{Consent for publication}

Not applicable.

\section{Competing interests}

The authors declare that they have no competing interests.

\section{Author details}

'Centre for Global Child Health, Hospital for Sick Children, Toronto, ON M5G OA4, Canada. ${ }^{2}$ Dalla Lana School of Public Health, University of Toronto, Toronto, Canada. ${ }^{3}$ Division of Adolescent Medicine, Hospital for Sick Children, Toronto, Canada. ${ }^{4}$ UNICEF, New York, USA. ${ }^{5}$ Spark Consulting, New York, USA. ${ }^{6}$ Center of Excellence in Women and Child Health, the Aga Khan University, Karachi, Pakistan

Received: 9 April 2020 Accepted: 30 November 2020

Published online: 14 December 2020

\section{References}

1. World Health Organization. Preventing Chronic Diseases - A Vital Investment: WHO Global Report. Geneva: World Health Organization; 2005

2. World Health Organization. Health for the world's adolescents: a second chance in the second decade. Geneva: World Health Organization; 2014

3. Sawyer SM, Afifi RA, Bearinger LH, Blakemore SJ, Dick B, Ezeh AC, et al. Adolescence: A foundation for future health. Lancet. 2012;379(9826):163040.

4. NCD Alliance. A focus on children and non-communicable diseases (NCDs). 2011.

5. Gore FM, Bloem PJN, Patton GC, Ferguson J, Joseph V, Coffey C, et al. Global burden of disease in young people aged 10-24 years: a systematic analysis. Lancet. 2011;377(9783):2093-102.

6. Beaglehole R, Bonita R, Horton R, Adams C, Alleyne G, Asaria P, et al. Priority actions for the non-communicable disease crisis. Lancet. 2011;377(9775): 1438-47.

7. Delisle H. Programming of chronic disease by impaired fetal nutrition: evidence and implications for research and intervention strategies. 2002

8. World Health Organization. Global health risks: mortality and burden of disease attributable to selected major risks. Geneva: World Health Organization; 2009

9. Ng M, Fleming T, Robinson M, Thomson B, Graetz N, Margono C, et al. Global, regional, and national prevalence of overweight and obesity in children and adults during 1980-2013: A systematic analysis for the global burden of disease study 2013. Lancet. 2014;384(9945):766-81.

10. Reilly JJ, Kelly J. Long-term impact of overweight and obesity in childhood and adolescence on morbidity and premature mortality in adulthood: systematic review. Int J Obes. 2011;35(7):891-8.

11. Lassi ZS, Salam RA, Das JK, Wazny K, Bhutta ZA. An unfinished agenda on adolescent health: opportunities for interventions. Semin Perinatol. 2015; 39(5):353-60.

12. Patton GC, Sawyer SM, Santelli JS, Ross DA, Afifi R, Allen NB, et al. Our future: a lancet commission on adolescent health and wellbeing. Lancet. 2016;387(10036):2423-78
13. Bennett JE, Stevens GA, Mathers CD, Bonita R, Rehm J, Kruk ME, et al. NCD countdown 2030: worldwide trends in non-communicable disease mortality and progress towards sustainable development goal target 3.4. Lancet. 2018;392(10152):1072-88.

14. WHO. Third UN High-level Meeting on Non-communicable Diseases New York. 2018;(September).

15. World Health Organization. 'BEST BUYS' and other recommended interventions for the prevention and control of noncommunicable diseases. 2017;18.

16. World Health Organization. Globbal Strategy to Reduce the Harmful Use of Alcohol. Geneva: World Health Organization; 2010.

17. World Health Organization. WHO Report on Global Tobacco Epidemic 2017: Global strategy to reduce the harmful use of alcohol. Geneva: World Health Organization; 2017.

18. World Health Organization. Taking Action on Childhood Obesity. Geneva: World Health Organization; 2018.

19. World Health Organization. NCD Global Monitoring Framework. Geneva: World Health Organization; 2013.

20. Pushka P. The vision and road map for addressing NCDs. In: International Conference on Healthy Lifestyles and NCDs in the Arab World and the Middle-East. Riyadh, KSA; 2012.

21. Blum RW, Astone NM, Decker MR, Mouli VC. A conceptual framework for early adolescence: a platform for research. Int J Adolesc Med Health. 2014; 26(3):321-31.

22. Viner RM, Ozer EM, Denny S, Marmot M, Resnick M, Fatusi A, et al. Adolescence and the social determinants of health. Lancet. 2012;379(9826): $1641-52$.

23. USAID. Multi-Sectoral Nutrition Strategy 2014-2015. 2014.

24. Black RE, Victora CG, Walker SP, Bhutta ZA, Christian P, De Onis M, et al. Maternal and child undernutrition and overweight in low-income and middle-income countries. Lancet. 2013;382(9890):427-51.

25. World Health Organization. Global Maternal, Newborn, Child and Adolescent Health Policy Indicator Database. 2014

26. World Health Organization. Global Health Observatory Data Repository. 2016

27. UNICEF. The State of the World's Children. Statistical Tables. 2017:2017.

28. The World Bank. Unemployment, youth total (\% of total labor force ages 15-24) (modeled ILO estimate). International Labour Organization, ILOSTAT database. Data retrieved in November 2017. 2017.

29. United Nations, Department of Economic and Social Affairs PD. World Population Prospects: The 2015 Revision. 2015.

30. Institute for Health Metrics and Evaluation. GBD Arrow Diagram. Seattle: Institute for Health Metrics and Evaluation; 2015.

31. Elgar FJ, Pförtner TK, Moor I, De Clercq B, Stevens GWJM, Currie C Socioeconomic inequalities in adolescent health 2002-2010: a time-series analysis of 34 countries participating in the health behaviour in school-aged children study. Lancet. 2015;385(9982):2088-95.

32. Elgar FJ, Craig W, Boyce W, Morgan A, Vella-Zarb R. Income inequality and school bullying: multilevel study of adolescents in 37 countries. J Adolesc Health. 2009;45(4):351-9.

33. Miyamoto K, Chevalier A. Education and health. In: Improving health and SOcial Cohension through education. Paris: OECD Publishing; 2010. p. 111-79.

34. Patel V, Flisher AJ, Hetrick S, McGorry P. Mental health of young people: a global public-health challenge. Lancet. 2007;369(9569):1302-13.

35. Gakidou E, Cowling K, Lozano R, Murray CJ. Increased educational attainment and its effect on child mortality in 175 countries between 1970 and 2009: a systematic analysis. Lancet. 2010;376(9745):959-74.

36. Patton GC, Sawyer SM, Santelli JS, Ross DA, Afi R, Allen NB, et al. Our future: a Lancet commission on adolescent health and wellbeing. 2016;6736(16).

37. International Labour Organization. Global Employment Trends for Youth 2013: A generation at risk. Geneva; 2013.

38. World Bank Group. GNI per capita, Atlas method (current US\$). World Bank national accounts data, and OECD National Accounts data files. 2018.

39. Victora CG, Bahl R, Barros AJD, França GVA, Horton S, Krasevec J, et al. Breastfeeding in the 21st century: epidemiology, mechanisms, and lifelong effect. Lancet. 2016:387(10017):475-90.

40. Kelishadi R, Farajian S. The protective effects of breastfeeding on chronic noncommunicable diseases in adulthood: A review of evidence. Adv Biomed Res. 2014;3:3 Available from: https:/pubmed.ncbi.nlm.nih.gov/24600594.

41. Brumana L, Arroyo A, Schwalbe NR, Lehtimaki S, Hipgrave DB. Maternal and child health services and an integrated, life-cycle approach to the 
prevention of non-communicable diseases. BMJ Glob Heal. 2017;2(3): e000295 Available from: https://pubmed.ncbi.nlm.nih.gov/29082005.

42. Rhodes $T$, Singer M, Bourgois P, Friedman SR, Strathdee SA. The social structural production of HIV risk among injecting drug users. Soc Sci Med. 2005;61(5):1026-44.

43. Amaugo LG, Papadopoulos C, Ochieng BMN, Ali N. The effectiveness of HIV/ AIDS school-based sexual health education programmes in Nigeria: a systematic review. Health Educ Res. 2014;29(4):633-48.

44. Blank L, Baxter SK, Payne N, Guillaume LR, Squires H. Systematic review and narrative synthesis of the effectiveness of contraceptive service interventions for young people, delivered in health care settings. Health Educ Res. 2012;27(6):1102-19.

45. Fonner VA, Armstrong KS, Kennedy CE, O'Reilly KR, Sweat MD. School based sex education and HIV prevention in low- and middle-income countries: A systematic review and meta-analysis. PLoS One. 2014;9(3):e89692.

46. Harrison A, Newell M-L, Imrie J, Hoddinott G. HIV prevention for south African youth: which interventions work? A systematic review of current evidence. BMC Public Health. 2010;10(1):102.

47. A MKSRG. The efficacy of interventions to reduce adolescent childbearing in low- and middle-income countries: a systematic review. TT -Stud Fam Plann. 2013;44(4):369-88.

48. Paul-Ebhohimhen VA, Poobalan A, Van Teijlingen ER. A systematic review of school-based sexual health interventions to prevent STI/HIV in sub-Saharan Africa. BMC Public Health. 2008;8.

49. Speizer IS, Magnani RJ, Colvin CE. The effectiveness of adolescent reproductive health interventions in developing countries: a review of the evidence. J Adolesc Health. 2003;33(5):324-48.

50. Hawkins JD, Catalano RF, Kosterman R, Abbott R, Hill KG. Preventing adolescent health-risk behaviors by strengthening protection during childhood. Arch Pediatr Adolesc Med. 1999;153(3):226-34.

51. Hawkins JD, Guo J, Hill KG, Battin-Pearson S, Abbott RD. Long-term effects of the Seattle social development intervention on school bonding trajectories. Appl Dev Sci. 2001;5(4):214-24.

52. Hawkins JD, Kosterman R, Catalano RF, Hill KG, Abbott RD. Promoting positive adult functioning through social development intervention in childhood. Arch Pediatr Adolesc Med. 2005;159(1):25-31.

53. Carson KV, Brinn MP, Labiszewski NA, Esterman AJ, Chang AB, Smith BJ. Community interventions for preventing smoking in young people. Cochrane Database Syst Rev. 2000;7:2-4.

54. Hawkins JD, Kosterman R, Catalano RF, Hill KG. \& Abbott RD. effects of social development intervention in childhood 15 years later. Arch Pediatr Adolesc Med. 2008;162(12):1133-41.

55. Lonczak HS, Abbott RD, Hawkins JD, Kosterman R, Catalano RF. Effects of the Seattle social development project on sexual behavior, pregnancy, birth, and sexually transmitted disease outcomes by age 21 years. Arch Pediatr Adolesc Med. 2002;156(5):438.

56. Cusimano MD, Sameem M. The effectiveness of middle and high schoolbased suicide prevention programmes for adolescents: a systematic review. Inj Prev. 2011;17(1):43-9.

57. Gould MS, Greenberg T, Velting DM, Shaffer D. Youth suicide risk and preventive interventions: a review of the past 10 years. J Am Acad Child Adolesc Psychiatry. 2003;42(4):386-405.

58. Katz C, Bolton SL, Katz LY, Isaak C, Tilston-Jones T, Sareen J. A systematic review of school-based suicide prevention programs. Depress Anxiety. 2013; 30(10):1030-45.

59. Klimes-Dougan B, Klingbeil DA, Meller SJ. The impact of universal suicideprevention programs on the help-seeking attitudes and behaviors of youths. Crisis. 2013;34(2):82-97.

60. Robinson J, Cox G, Malone A, Williamson M, Baldwin G, Fletcher K, et al. A systematic review of school-based interventions aimed at preventing, treating, and responding to suicide- related behavior in young people. Crisis. 2013;34(3):164-82.

61. World Health Organization. Adolescent mental health: mapping actions of nongovernmental organizations and other international development organizations. Geneva; 2012.

62. Mason-jones AJ, Crisp C, Momberg M, Koech J, Koker P De, Mathews C. A systematic review of the role of school-based healthcare in adolescent sexual, reproductive, and mental health A systematic review of the role of school-based healthcare in adolescent sexual, reproductive, and mental health. 2012;

63. Owen J, Carroll C, Cooke J, Formby E, Hayter M, Hirst J, et al. School-linked sexual health services for young people (SSHYP): A survey and systematic review concerning current models, effectiveness, cost-effectiveness and research opportunities. Health Technol Assess. 2010;14(30):1-228, iii-iv.

64. Foxcroft DR, Tsertsvadze A. Universal family-based prevention programs for alcohol misuse in young people. Cochrane Libr. 2011.

65. Binagwaho A, Wagner CM, Gatera M, Karema C, Nutt CT, Ngabo F. Achieving high coverage in Rwanda's national human papillomavirus vaccination programme. Bull World Health Organ. 2012:623-8.

66. LaMontagne DS, Barge S, Thi Le N, Mugisha E, Penny ME, Gandhi S, Janmohamed A, Kumakech E, Mosquiera NR, Nguyen NQ, Paul P, Tang Y, Minh TH, Uttekar BP, Jumaan AO. Human papillomavirus vaccine delivery strategies that achieved high coverage in low- and middle-income countries. Bull World Health Organ. 2011;89(11):821-30.

67. Ladner J, Besson MH, Rodrigues M, Audureau E, Saba J. Performance of 21 HPV vaccination programs implemented in low and middle-income countries, 2009-2013. BMC Public Health. 2014;14:1-11.

68. Haider R. Adolescent nutrition: a review of the situation in selected southeast Asian countries. New Delhi; 2006.

69. Catalano RF, Fagan AA, Gavin LE, Greenberg MT, Irwin CE, Ross DA, et al. Worldwide application of prevention science in adolescent health. Lancet. 2012;379(9826):1653-64.

70. Botvin GJ, Griffin KW, Nichols TD. Preventing youth violence and delinquency through a universal school-based prevention approach. Prev Sci. 2006;7(4):403-8.

71. Botvin GJ, Griffin KW. Life skills training: empirical findings and future directions. J Prim Prev. 2004;25(2):211-32.

72. DiClemente RJ, Wingood GM, Harrington KF, Lang DL, Davies SL, lii EWH, et al. Efficacy of an HIV prevention intervention. J Am Med Assoc. 2004; 292(2):171-9.

73. Elliot DS. Blueprints for violence prevention. Boulder; 1998.

74. Faggiano F, Vigna-Taglianti F, Burkhart G, Bohrn K, Cuomo L, Gregori D, et al. The effectiveness of a school-based substance abuse prevention program: 18-month follow-up of the EU-dap cluster randomized controlled trial. Drug Alcohol Depend. 2010;108(1-2):56-64.

75. Gottschalk LB, Ortayli N. Interventions to improve adolescents' contraceptive behaviors in low- and middle-income countries: a review of the evidence base. Contraception. 2014;90(3):211-25.

76. Griffin KW, Botvin GJ, Nichols TR. Long-term follow-up effects of a schoolbased drug abuse prevention program on adolescent risky driving. Prev Sci. 2004;5(3):207-12.

77. Fenton KA, Johnson AM, Mcmanus S, Erens B. Series editors measuring sexual behaviour : methodological challenges in survey research. Sex transm Inf. 2001:84-92

78. Kirby D, Obasi A, Laris BA. The effectiveness of sex education and HIV education interventions in schools in developing countries. World Heaolth Organ Tech Rep Ser. 2006;938(103):317-41.

79. Campbell FA, Ramey CT, Miller-johnson S. Early childhood education : young adult outcomes from the abecedarian project Elizabeth Pungello and Joseph sparling. Appl Dev Sci. 2002;6(1):42-57.

80. Fagan AA, Catalano RF. What works in youth violence prevention: a review of the literature. Res Soc Work Pract. 2013;23(2):141-56.

81. Gates S, Mccambridge J, Smith LA, Foxcroft D. Interventions for prevention of drug use by young people delivered in non-school settings. Cochrane Database Syst Rev. 2009;1.

82. Kumpfer KL, Alvarado $\mathrm{R}$, Whiteside HO. Family-based interventions for substance use and misuse prevention. Subst Use Misuse. 2003;38(11-13):1759-87 1916.

83. Controlled R, Olds D, Henderson CR, Cole R, Eckenrode J, Kitzman H, et al. Long-term Effects of Nurse Home Visitation on Children 's Criminal and Antisocial Behavior. 2014;280(14).

84. Olds DL, Kitzman H, Cole R, Robinson J, Sidora K, Luckey DW, Henderson CR, Hanks C, Bondy J, Holmberg J. Effects of nurse home-visiting on maternal life course and child development: age 6 follow-up results of a randomized trial. Pediatrics. 2004;114(6):1550-9.

85. Olds DL, Robinson J, O'Brien R, Luckey DW, Pettitt LM, Henderson CR, Ng RK, Sheff KL, Korfmacher J, Hiatt S, Talmi A. Home visiting by paraprofessionals and by nurses: a randomized, controlled trial. Pediatrics. 2002;110(3):486-96.

86. Martineau F, Tyner E, Lorenc T, Petticrew M, Lock K. Population-level interventions to reduce alcohol-related harm: an overview of systematic reviews. Prev Med (Baltim). 2013;57(4):278-96.

87. Reynolds AJ, Temple JA, Ou S-R, Robertson DL, Mersky JP, Topitzes JW, et al. Effects of a school-based, early childhood intervention on adult health and well-being. Arch Pediatr Adolesc Med. 2007;161(8):730. 
88. Reynolds AJ, Temple JA, Robertson DL, Mann EA. Long-term effects of an early childhood intervention on educational achievement and juvenile arrest: A 15-year follow-up of low-income children in public schools. JAMA. 2001;285(18):2339-46.

89. Schinke SP, Schwinn TM, Noia J, Cole KC. Reducing the risks of alcohol use among urban youth: three-year effects of a computer-based intervention with and without parent involvement. J Stud Alcohol. 2004;65(4):443-9.

90. Schweinhart LJ, Barnes HV, Weikhart DP. Significant benefits: The High Scope Perry preschool study through age 27. In: Child Welfare: Major Themes in Health and Social Welfare. London; New York: Routledge; 2005. p. 9-29.

91. Schwinn TM, Schinke SP. Preventing alcohol use among late adolescent urban youth: 6-year results from a computer-based intervention. J Stud Alcohol Drugs. 2010;71(4):535-8.

92. Spoth R, Trudeau L, Guyll M, Shin C, Redmond C. Universal intervention effects on substance use among young adults mediated by delayed adolescent substance initiation. J Consult Clin Psychol. 2009;77(4):620-32.

93. Thomas RE, Baker PR, Thomas BC, Lorenzetti DL. Family-based programmes for preventing smoking by children and adolescents. Cochrane Database Syst Rev. 2015;2015(2):CD004493.

94. Trickett EJ, Beehler S. The ecology of multilevel interventions to reduce social inequalities in health. Am Behav Sci. 2013;57(8):1227-46.

95. Gavin LE, Catalano RF, David-Ferdon C, Gloppen KM, Markham CM. A review of positive youth development programs that promote adolescent sexual and reproductive health. J Adolesc Health. 2010;46(3 SUPPL):S75-91.

96. Faggiano F, Minozzi S, Versino E, Buscemi D, Faggiano F, Minozzi S, et al. Universal school-based prevention for illicit drug use ( review ). Cochrane Database Syst Rev. 2016;3(12):1-67.

97. Thomas RE, Lorenzetti DL, Spragins W. Systematic review of mentoring to prevent or reduce tobacco use by adolescents. Acad Pediatr. 2013;13(4):300-7.

98. Sethi, D., Hughes, K., Bellis, M., Mitis, F., \& Racioppi F. European report on preventing violence and knife crime among young people. 2010.

99. Ellsberg M, Arango DJ, Morton M, Gennari F, Kiplesund S, Contreras M, et al. Prevention of violence against women and girls: what does the evidence say? Lancet. 2015;385(9977):1555-66.

100. Brittain AW, Williams JR, Zapata LB, Pazol K, Romero LM, Weik TS. Youthfriendly family planning Services for Young People: a systematic review. Am J Prev Med. 2015:49(2):S73-84.

101. Hale DR, Fitzgerald-Yau N, Viner R. A systematic review of effective interventions for reducing multiple health risk behaviors in adolescence. Am J Public Health. 2014;104(5):e19-41.

102. World Health Organization. Violence prevention: the evidence. Geneva; 2010

103. Pettifor A, MacPhail C, Nguyen N. \& RM. can money prevent the spread of HIV? A review of cash payments for HIV prevention. AIDS Behav. 2012;16(7):1729-38.

104. Wamoyi J, Mshana G, Mongi A, et al. A review of interventions addressing structural drivers of adolescents' sexual and reproductive health vulnerability in sub-Saharan Africa: implications for sexual health programming. Reprod Heal. 2014;11:88.

105. Baird SJ, Garfein RS, McIntosh CT, Özler B. Effect of a cash transfer programme for schooling on prevalence of HIV and herpes simplex type 2 in Malawi: a cluster randomised trial. Lancet. 2012;379(9823):1320-9.

106. Zuurmond MA, Geary RS, Ross DA. The effectiveness of youth centers in increasing use of sexual and reproductive health services: a systematic review. Stud Fam Plan. 2012;43(4):239-54.

107. Lundgren $\mathrm{R}$, Amin A. Addressing intimate partner violence and sexual violence among adolescents: emerging evidence of effectiveness. J Adolesc Health. 2015;56(1):S42-50.

108. Allen-Meares P, Montgomery KL, Kim JS. School-based social work interventions: a cross-national systematic review. Soc Work (United States). 2013;58(3):253-62.

109. Pearson N, Braithwaite R, Biddle S. The effectiveness of interventions to increase physical activity among adolescent girls: a meta-analysis. Acad Pediatr. 2015;15(1):9-18.

110. Duflo, E., Dupas, P., Kremer, M., \& Sinei S. Education and HIV/AIDS prevention: evidence from a randomized evaluation in Western Kenya. 2006 (World Bank Policy Research Working Paper). Report No.: 4024

111. Fiszbein, A. \& Schady N. Conditional Cash Transfers: Reducing Present and Future Poverty. 2009

112. Baird SJ, Garfein RS, Mclntosh CT, Özler B. The short-term impacts of a schooling conditional cash transfer program on the sexual behavior of young women. Health Econ. 2010;19(S1):55068.
113. Moyer-Gusé E. Toward a theory of entertainment persuasion: explaining the persuasive effects of entertainment-education messages. Commun Theory. 2008;18(3):407-25

114. Buse K, Tanaka S, Hawkes S. Healthy people and healthy profits? Elaborating a conceptual framework for governing the commercial determinants of non-communicable diseases and identifying options for reducing risk exposure. Glob Health. 2017;13(1):1-12.

115. Moodie R, Stuckler D, Monteiro C, Sheron N, Neal B, Thamarangsi T, et al. Profits and pandemics: prevention of harmful effects of tobacco, alcohol, and ultra-processed food and drink industries. Lancet. 2013;381(9867):670-9.

116. United Nations. Human Rights Council, 26th Session Al 3. Grover A. Report of the Special Rapporteur on the right of everyone to the enjoyment of the highest attainable standard of physical and mental health. United Nations: 2014 p. A/HRC/26/31.

117. Atun R, Jaffar S, Nishtar S, Knaul FM, Barreto ML, Nyirenda M, et al. Improving responsiveness of health systems to non-communicable diseases. Lancet. 2013;381(9867):690-7.

118. Celletti F, Wright A, Palen J, Frehywot S, Markus A, Greenberg A, et al. Can the deployment of community health workers for the delivery of HIV services represent an effective and sustainable response to health workforce shortages? Results of a multicountry study. AIDS. 2010;24(Suppl 1):S45-57.

119. Kalk A, Groos N, Karasi JC, Girrbach E. Health systems strengthening through insurance subsidies: the GFATM experience in Rwanda. Trop Med Int Heal. 2010;15(1):94-7.

120. Harrison K, Rahimi N, Carolina D-HM. Factors limiting data quality in the expanded programme on immunization in low and middle-income countries: a scoping review. Vaccine. 2020;38(30):4652-63 Available from: http://www.sciencedirect.com/science/article/pii/S0264410X20304011.

\section{Publisher's Note}

Springer Nature remains neutral with regard to jurisdictional claims in published maps and institutional affiliations.

Ready to submit your research? Choose BMC and benefit from:

- fast, convenient online submission

- thorough peer review by experienced researchers in your field

- rapid publication on acceptance

- support for research data, including large and complex data types

- gold Open Access which fosters wider collaboration and increased citations

- maximum visibility for your research: over $100 \mathrm{M}$ website views per year

At $\mathrm{BMC}$, research is always in progress.

Learn more biomedcentral.com/submissions 